\title{
Cross-layer and Reliable Opportunistic Routing Algorithm for Mobile Ad Hoc Networks
}

\author{
Ning Li, student member, IEEE, Jose-Fernan Martinez-Ortega, Vicente Hernandez Diaz
}

\begin{abstract}
For improving the efficiency and the reliability of the opportunistic routing algorithm, we propose a cross-layer and reliable opportunistic routing algorithm (CBRT) for Mobile Ad Hoc Networks, in which the fuzzy logic and topology control are introduced into the opportunistic routing algorithm. In CBRT, the inputs of the fuzzy logic system are the relative variances ( $r v)$ of the metrics rather than the metrics' values; by this, the number of fuzzy rules is reduced dramatically. Moreover, the number of fuzzy rules does not increase when the number of inputs increases. For improving routing reliability and reducing the control cost, in CBRT, the relay node degree is a range rather than a constant number. The nodes are divided into different categories based on their relay node degree. The nodes adjust their transmission power based on which categories that they belong to. Additionally, for investigating the effect of the node mobility on routing performance, we propose a link lifetime prediction algorithm which takes the moving speed and moving direction into account. In CBRT, the source node determines the relaying priorities of the candidate relay nodes based on their utilities. The relay node whose utility is large has high priority to forward the data packet to next hop. By these innovations, the performance of CBRT is much better than that of ExOR; however, the computation complexity does not increase in CBRT.
\end{abstract}

Index Terms-Cross-layer, fuzzy logic, topology control, opportunistic routing.

\section{INTRODUCTION}

The Mobile Ad Hoc Networks (MANETs) are widely used in the Internet of Things (IoT), such as in battlefield [1][2], vehicle networks [3][4][5], underwater cooperation robot networks [6][7][8], etc. In MANETs, one of the important issues is the routing protocol design, which guarantees reliable and efficient data transmission from source node to destination node. There are two routing strategies for the MANETs: deterministic routing and opportunistic routing [9]. In deterministic routing, the sender sends data packet to one neighbor which is chosen based on the optimal algorithms; in opportunistic routing, the sender sends the data packet to a set of relay nodes rather than only one relay node to improve the packet delivery ratio between sender and receiver $[10][11][12][13]$. In this paper, we mainly focus on the opportunistic routing.

\section{A. Motivations}

Ning Li, Jose-Fernan Martinez-Ortega, and Vecente Hernandez Diaz are with the Universidad Politenica de Madrid, Madrid, Spain.

The research leading to the presented results has been undertaken with in the SWARMs European project (Smart and Networking Underwater Robots in Cooperation Meshes), under Grant Agreement n. 662107-SWARMs-ECSEL -2014-1, partially supported by the ECSEL JU and the Spanish Ministry of Economy and Competitiveness (Ref: PCIN-2014-022-C02-02).

E-mail: \{li.ning, jf.martinez, vicente.hernandez\}@upm.es.
In opportunistic routing, the source node chooses the candidate relay set (CRS) [13] and decides the priorities of the relay nodes based on specific metrics, such as the distance to the destination node, the expected transmission count (ETX), the propagation delay, the packet queue length, etc. [13]. These performance metrics are relevant and interplay; the more performance metrics are taken into account, the better routing performance is [14]. In traditional opportunistic routing algorithm, since the source node chooses the relay nodes based on a limited number of metrics, so it cannot reflect the comprehensive characteristics of the node. For instance, when the source node chooses and prioritizes the relaying nodes based on their ETX, the nodes whose ETX is small will be chosen and set with high priorities. However, considering the parameters in MAC layer, link layer, or physic layer, the node which the ETX is small does not mean that the other parameters are also optimal. For finding the tradeoff between different cross-layer parameters, the fuzzy logic has been introduced into the routing algorithm design, such as in [14], [15], [16], [17], and [18], etc. However, the main disadvantage of fuzzy logic is that when the number of inputs increases, the number of fuzzy rules increases exponentially, which will be proved in Section III. This will limit the future performance improvement in the fuzzy logic based opportunistic routing algorithms. In general, at the moment, the common number of inputs is no larger than 3 [14-18]. So one of the purposes in this paper is how to reduce the computation complexity of the fuzzy logic system that caused by the increase of the inputs' number.

In opportunistic routing, the more nodes in CRS, the higher packet delivery ratio between the sender and the CRS is; vice versa. However, due to the mobility of the nodes in MANETs, the number of neighbors changes frequently, so the packet delivery ratio between the sender and the CRS changes frequently, too. In case there are too few or too many relay nodes in CRS, we introduce the topology control, which is good at controlling the number of neighbors [19], into the opportunistic routing algorithm. However, due to the high mobility of nodes in MANETs, if each node maintains a constant number of nodes in CRS, the transmission power needs to be adjusted frequently, which will consume a plenty of network resource that could have been used in data packet transmission. So the topology control algorithm should be able to reduce the extra control cost as far as possible. Moreover, since the node mobility has great effect on the routing performance, so the node mobility, including the moving speed and moving direction, should also be taken into account during the routing algorithm design.

\section{B. Main contributions}


Motivated by these, we propose the cross-layer and reliable opportunistic routing algorithm (CBRT) for MANETs in this paper. In CBRT, the inputs of the fuzzy logic system are the relative variances $(r v)$ of the metrics rather than the metrics' values, which can reduce the number of fuzzy rules dramatically. Moreover, the number of fuzzy rules does not increase when the number of inputs increases. For reducing the control cost, the number of nodes in CRS is a range rather than a constant number. The nodes are divided into different categories based on the numbers of nodes in their CRS. The nodes adjust their transmission power based on which categories that they belong to. The source node determines the priorities of the relay nodes in CRS based on the utilities of these nodes. The relay node which the utility is large has high relaying priority. The main contributions of this paper are summarized as follows:

- We propose a scatter based fuzzy logic algorithm (SBFL) which can handle multiple cross-layer parameters at the same time without increasing the computation complexity; to the best of our knowledge, this is the first algorithm which can take multiple cross-layer parameters into account without increasing the computation complexity seriously.

- We propose a link lifetime prediction algorithm which takes both the moving speed and the moving direction into account to improve the accuracy;

- We propose a packet delivery ratio based opportunistic topology control algorithm for maintaining stable node number in CRS; in this algorithm, the node number in CRS is stable and the transmission power adjustment ratio is low;

- Based on the topology control algorithm and SBFL algorithm, we propose the cross-layer balanced and reliable opportunistic routing algorithm, which utilizes both the fuzzy logic and topology control to improve the routing performance.

The remaining of this paper organized as follows: in Section 2, we introduce the related works; Section 3 introduces the principle of SBFL; the link lifetime prediction algorithm and the packet delivery ratio based topology control algorithm are presented in Section 4; moreover, the CBRT algorithm is also introduced in Section 4; Section 5 evaluates the performance of the SBFL algorithm and the CBRT algorithm, and compares the CBRT algorithm with the ExOR algorithm; Section 6 concludes the work in this paper.

\section{RELATED WORKS}

Since the opportunistic routing has been proposed by Sanjit Biswas and Robert Morris [20], many opportunistic routing algorithms have been developed in recent years. In [21], the authors propose a relaying node selection algorithm for a 1-D wireless sensor network, named energy saving via opportunistic routing (ENS_OR). The ENS_OR selects the candidate relays set and prioritizes the nodes in it based on their virtual optimal transmission distance and residual energy level. The nodes which are closer to the energy equivalent nodes and have more residual energy than the source node will be selected as the relaying nodes. In [22], the authors propose two accurate energy consumption based objective functions, which exploit the knowledge of both the frame error ratio within the physical layer, the maximum number of retransmissions in the medium access control (MAC), and the number of relays in the network layer. Based on the objective functions, the routing algorithm is designed for opportunistic routing, which employs the objective functions to prioritize the nodes in the candidate relays set. The authors in [23] exploit the geographic opportunistic routing (GOR) for Quality of Services (QoS) provisioning with both the end to end reliability and delay constraints in wireless sensor networks (WSNs); moreover, the authors also define the problem of efficient GOR for multiconstrained QoS provisioning in WSNs. Based on these conclusions, the authors propose the Efficient QoS-aware GOR protocol for the WSNs, in which the energy efficiency, latency, and the time complexity are taken into account to select the relaying nodes. The authors in [24] propose a distributed optimal community-aware opportunistic routing algorithm, in which the home-aware community model is proposed. In this algorithm, first, the routing between lots of nodes are turned to the routing between a few community homes; then the algorithm maintains an optimal candidate relays set for each home. Each home only forwards its message to the node in its optimal candidate relays set. More opportunistic routings can be found in [25], [26] and [13]; especially in [13], the author reviews the opportunistic routing algorithms in recent years and identifies and discusses the future research directions related to the opportunistic routing design, optimization, and deployment.

Recently, the fuzzy logic becomes popular in routing protocols design. In [27], the authors propose an energy-effective cross-layer routing protocol for WSNs based on fuzzy logic. In this protocol, for minimizing the energy consumption and maximizing the network lifetime, the algorithm takes the remaining battery reserve capacity, the link quality, and the transmission power of the neighbor nodes into account to select the next hop relaying nodes. In [28], for reducing the average end-to-end delay of the MANETs, the authors propose a fuzzy logic based adaptive cross-layer routing protocol for the delay-sensitive applications. In this algorithm, each node can switch between reactive routing mode and proactive routing mode based on the current node status separately. The algorithm uses the fuzzy logic controller to decide the routing mode of each node. In [29], the authors propose a routing algorithm for WSNs which can extend the network lifetime and balance the energy consumption by combining the fuzzy approach and the A-star algorithm together. The remaining battery power, the number of hops to the destination node, and the traffic loads are taken into consideration to determine the optimal routing path from the source node to the destination node. Similar in [30], to prolong the network lifetime, a fuzzy logic based energy-optimization routing protocol is proposed, in which the social welfare function is used to predict inequality of residual energy of 
neighbor nodes. The algorithm computes the degree of energy balance based on the energy inequality. The fuzzy logic system uses the degree of node closeness to the shortest path, the degree of node closeness to sink, and the degree of energy balance to achieve the routing decision. Additionally, in [31], the node density, the delay, and the number of dead nodes are used as inputs of the fuzzy logic system to select the next hop relaying node for achieving the balanced energy consumption of all the nodes with minimum delay. More related works about the fuzzy logic based routing algorithms can also be found in [32], [33], [34], and [35].

Recently, more and more researchers consider that integrate the topology control into the routing algorithm in wireless sensor network or ad hoc network is an effective way to improve the network performance. In [36], the authors proposed three alternative mathematical models for integrating topology control and routing decisions so as to prolong the lifetime of sensors. For reducing the effect of primary user (PU) activities and node mobility to the stability of links in mobile cognitive networks, in [37], the authors proposed a primary user activity prediction based joint topology control and stable routing protocol (PP-JTCSR), which can quantitatively capture channel utilization patterns of PUs. By topology control in PP-JTCSR, the most stable and shortest path can be found. In [38], the authors investigate the impact of two topology control methods for resolving the problem of void/isolated nodes appeared in geographic routing protocols, which can reduce the number of void/isolated nodes significantly. In [39], the authors have shown that joint topology control and routing assignment as an optimization problem is a NP-hard problem. For solving this problem, in this paper, the author proposed TORA (joint topology control and routing assignment) which seeks to jointly optimize topology and routing for DMesh (directional antennas in wireless mesh networks). There are also many researchers that combine the routing design with the topology control, which can be found in [40], [41], [42], and [43].

\section{PRINCIPLE OF THE SCATTER BASED FUZZY LOGIC ALGORITHM}

In this section, the principle of scatter based fuzzy logic algorithm (SBFL) will be introduced in detail.

\section{A. Problem statement}

As shown in Figure 1, the fuzzy logic system is divided into three modules: fuzzification, fuzzy logic inference, and defuzzification.

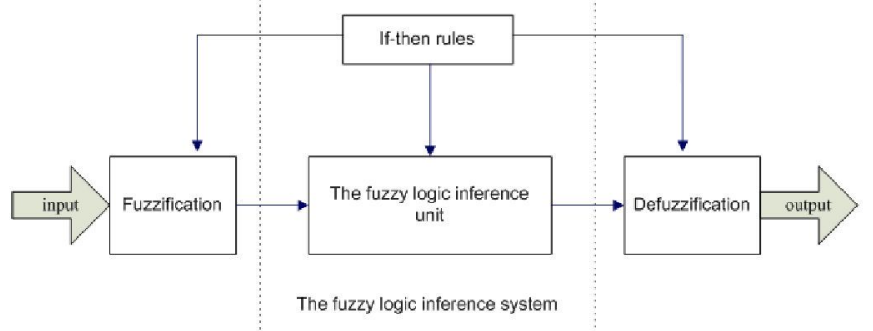

Fig. 1. The process of fuzzy logic system
In fuzzification module, the input variables are mapped to fuzzy set based on linguistic variables and membership functions. Assuming that the input universe is $U$ and the fuzzy set of universe $U$ is $A ; \mu_{A}$ is the membership function which maps universe $U$ to fuzzy set $A$. The membership function represents the membership between universe $U$ and fuzzy set $A$, i.e., $\mu_{A}: U \rightarrow A \in[0,1]$. The fuzzy set $A$ can be expressed as: $A=\left\{\left(u_{i}, \mu_{A}\left(u_{i}\right)\right) \mid u_{i} \in U\right\}$, where $u_{i}$ is the element of universe $U$. The general definition of the membership function is:

$$
\left\{\begin{array}{l}
\mu_{A}\left(u_{i}\right)=1: \quad u_{i} \text { belongs to A complete; } \\
\mu_{A}\left(u_{i}\right)=0: \quad u_{i} \text { does not belong to A complete } ; \\
0<\mu_{A}\left(u_{i}\right)<1: \quad u_{i} \text { belongs to A partly; }
\end{array}\right.
$$

The fuzzy set $A$ is the input of the fuzzy inference module which will be used to calculate the output fuzzy set $B$. The core part of the fuzzy inference system is the if-then rules (fuzzy rules). The if-then rules determine the relationship between the inputs (fuzzy set $A$ ) and outputs (fuzzy set $B$ ). The form of the fuzzy rule is generally expressed as:

$$
\text { If } A \text { (input) then } B \text { (output) }
$$

where $A$ and $B$ are the fuzzy sets of universe $X$ (input) and $Y$ (output), respectively. The membership functions are $\mu_{A}\left(x_{i}\right)$ and $\mu_{B}\left(y_{i}\right)$, where $x_{i} \in X, y_{i} \in Y$. Thus according to the fuzzy mathematics theory (Mamdani fuzzy inference algorithm), the membership function between fuzzy set $A$ and fuzzy set $B$ can be decided by:

$$
\mu_{A \rightarrow B}(x, y)=\mu_{R}(x, y) \stackrel{\Delta}{=} \mu_{A}(x) \wedge \mu_{B}(y)
$$

where $R$ represents the fuzzy relationship between universe $X$ and universe $Y$, i.e., $R=A \rightarrow B$; " $\wedge$ " is the conjunction operator in discrete mathematics. By (3), the fuzzy result can be got.

However, for taking more cross-layer performance metrics into account, the fuzzy rules will be much more complex than that shown in (2), which is a multi-inputs-single-output fuzzy system. In this scenario, the fuzzy rules will be:

$$
\text { If } A_{1}, A_{2}, A_{3}, \ldots, A_{n} \text { then } B
$$

where $A_{1}, A_{2}, A_{3}, \ldots, A_{n}$ (inputs) and $B$ (output) are the fuzzy sets of universes $U_{1}, U_{2}, U_{3}, \ldots . ., U_{n}$ (inputs) and $U$ (output), respectively. The membership functions are $\mu_{A_{1}}\left(u_{1}\right)$, $\mu_{A_{2}}\left(u_{2}\right), \quad \mu_{A_{3}}\left(u_{3}\right), \ldots \ldots, \mu_{A_{n}}\left(u_{n}\right), \quad \mu_{B}(u)$, where $u_{1} \in U_{1}$, $u_{2} \in U_{2}, u_{3} \in U_{3}, \ldots, u_{n} \in U_{n}, u \in U$. So the membership function can be expressed as:

$$
\begin{array}{r}
\mu_{\left\{A_{1}, A_{2}, A_{3}, \ldots . . A_{\left.A_{n}\right\}}\right\} B}\left(u_{1}, u_{2}, u_{3}, \ldots . ., u_{n}, u\right)=\mu_{R}\left(u_{1}, u_{2}, u_{3}, \ldots \ldots, u_{n}, u\right) \\
\stackrel{\Delta}{=} \mu_{A_{1}}\left(u_{1}\right) \wedge \mu_{A_{2}}\left(u_{2}\right) \wedge \mu_{A_{3}}\left(u_{3}\right) \cdots \wedge \mu_{A_{n}}\left(u_{n}\right) \wedge \mu_{B}(u)
\end{array}
$$

where $R=\left\{A_{1}, A_{2}, A_{3}, \ldots . ., A_{n}\right\} \rightarrow B$ is the fuzzy relationship between universe $U_{1}, U_{2}, U_{3}, \ldots ., U_{n}$ and $U$.

As shown in (5), in the multi-inputs-single-output fuzzy system, the number of the permutation and combination between the fuzzy sets are huge. The (5) illustrates that with 


\title{
Cross-layer and Reliable Opportunistic Routing Algorithm for Mobile Ad Hoc Networks
}

\author{
Ning Li, student member, IEEE, Jose-Fernan Martinez-Ortega, Vicente Hernandez Diaz
}

\begin{abstract}
For improving the efficiency and the reliability of the opportunistic routing algorithm, we propose a cross-layer and reliable opportunistic routing algorithm (CBRT) for Mobile Ad Hoc Networks, in which the fuzzy logic and topology control are introduced into the opportunistic routing algorithm. In CBRT, the inputs of the fuzzy logic system are the relative variances ( $r v)$ of the metrics rather than the metrics' values; by this, the number of fuzzy rules is reduced dramatically. Moreover, the number of fuzzy rules does not increase when the number of inputs increases. For improving routing reliability and reducing the control cost, in CBRT, the relay node degree is a range rather than a constant number. The nodes are divided into different categories based on their relay node degree. The nodes adjust their transmission power based on which categories that they belong to. Additionally, for investigating the effect of the node mobility on routing performance, we propose a link lifetime prediction algorithm which takes the moving speed and moving direction into account. In CBRT, the source node determines the relaying priorities of the candidate relay nodes based on their utilities. The relay node whose utility is large has high priority to forward the data packet to next hop. By these innovations, the performance of CBRT is much better than that of ExOR; however, the computation complexity does not increase in CBRT.
\end{abstract}

Index Terms-Cross-layer, fuzzy logic, topology control, opportunistic routing.

\section{INTRODUCTION}

The Mobile Ad Hoc Networks (MANETs) are widely used in the Internet of Things (IoT), such as in battlefield [1][2], vehicle networks [3][4][5], underwater cooperation robot networks [6][7][8], etc. In MANETs, one of the important issues is the routing protocol design, which guarantees reliable and efficient data transmission from source node to destination node. There are two routing strategies for the MANETs: deterministic routing and opportunistic routing [9]. In deterministic routing, the sender sends data packet to one neighbor which is chosen based on the optimal algorithms; in opportunistic routing, the sender sends the data packet to a set of relay nodes rather than only one relay node to improve the packet delivery ratio between sender and receiver $[10][11][12][13]$. In this paper, we mainly focus on the opportunistic routing.

\section{A. Motivations}

Ning Li, Jose-Fernan Martinez-Ortega, and Vecente Hernandez Diaz are with the Universidad Politenica de Madrid, Madrid, Spain.

The research leading to the presented results has been undertaken with in the SWARMs European project (Smart and Networking Underwater Robots in Cooperation Meshes), under Grant Agreement n. 662107-SWARMs-ECSEL -2014-1, partially supported by the ECSEL JU and the Spanish Ministry of Economy and Competitiveness (Ref: PCIN-2014-022-C02-02).

E-mail: \{li.ning, jf.martinez, vicente.hernandez\}@upm.es.
In opportunistic routing, the source node chooses the candidate relay set (CRS) [13] and decides the priorities of the relay nodes based on specific metrics, such as the distance to the destination node, the expected transmission count (ETX), the propagation delay, the packet queue length, etc. [13]. These performance metrics are relevant and interplay; the more performance metrics are taken into account, the better routing performance is [14]. In traditional opportunistic routing algorithm, since the source node chooses the relay nodes based on a limited number of metrics, so it cannot reflect the comprehensive characteristics of the node. For instance, when the source node chooses and prioritizes the relaying nodes based on their ETX, the nodes whose ETX is small will be chosen and set with high priorities. However, considering the parameters in MAC layer, link layer, or physic layer, the node which the ETX is small does not mean that the other parameters are also optimal. For finding the tradeoff between different cross-layer parameters, the fuzzy logic has been introduced into the routing algorithm design, such as in [14], [15], [16], [17], and [18], etc. However, the main disadvantage of fuzzy logic is that when the number of inputs increases, the number of fuzzy rules increases exponentially, which will be proved in Section III. This will limit the future performance improvement in the fuzzy logic based opportunistic routing algorithms. In general, at the moment, the common number of inputs is no larger than 3 [14-18]. So one of the purposes in this paper is how to reduce the computation complexity of the fuzzy logic system that caused by the increase of the inputs' number.

In opportunistic routing, the more nodes in CRS, the higher packet delivery ratio between the sender and the CRS is; vice versa. However, due to the mobility of the nodes in MANETs, the number of neighbors changes frequently, so the packet delivery ratio between the sender and the CRS changes frequently, too. In case there are too few or too many relay nodes in CRS, we introduce the topology control, which is good at controlling the number of neighbors [19], into the opportunistic routing algorithm. However, due to the high mobility of nodes in MANETs, if each node maintains a constant number of nodes in CRS, the transmission power needs to be adjusted frequently, which will consume a plenty of network resource that could have been used in data packet transmission. So the topology control algorithm should be able to reduce the extra control cost as far as possible. Moreover, since the node mobility has great effect on the routing performance, so the node mobility, including the moving speed and moving direction, should also be taken into account during the routing algorithm design.

\section{B. Main contributions}


Motivated by these, we propose the cross-layer and reliable opportunistic routing algorithm (CBRT) for MANETs in this paper. In CBRT, the inputs of the fuzzy logic system are the relative variances $(r v)$ of the metrics rather than the metrics' values, which can reduce the number of fuzzy rules dramatically. Moreover, the number of fuzzy rules does not increase when the number of inputs increases. For reducing the control cost, the number of nodes in CRS is a range rather than a constant number. The nodes are divided into different categories based on the numbers of nodes in their CRS. The nodes adjust their transmission power based on which categories that they belong to. The source node determines the priorities of the relay nodes in CRS based on the utilities of these nodes. The relay node which the utility is large has high relaying priority. The main contributions of this paper are summarized as follows:

- We propose a scatter based fuzzy logic algorithm (SBFL) which can handle multiple cross-layer parameters at the same time without increasing the computation complexity; to the best of our knowledge, this is the first algorithm which can take multiple cross-layer parameters into account without increasing the computation complexity seriously.

- We propose a link lifetime prediction algorithm which takes both the moving speed and the moving direction into account to improve the accuracy;

- We propose a packet delivery ratio based opportunistic topology control algorithm for maintaining stable node number in CRS; in this algorithm, the node number in CRS is stable and the transmission power adjustment ratio is low;

- Based on the topology control algorithm and SBFL algorithm, we propose the cross-layer balanced and reliable opportunistic routing algorithm, which utilizes both the fuzzy logic and topology control to improve the routing performance.

The remaining of this paper organized as follows: in Section 2, we introduce the related works; Section 3 introduces the principle of SBFL; the link lifetime prediction algorithm and the packet delivery ratio based topology control algorithm are presented in Section 4; moreover, the CBRT algorithm is also introduced in Section 4; Section 5 evaluates the performance of the SBFL algorithm and the CBRT algorithm, and compares the CBRT algorithm with the ExOR algorithm; Section 6 concludes the work in this paper.

\section{RELATED WORKS}

Since the opportunistic routing has been proposed by Sanjit Biswas and Robert Morris [20], many opportunistic routing algorithms have been developed in recent years. In [21], the authors propose a relaying node selection algorithm for a 1-D wireless sensor network, named energy saving via opportunistic routing (ENS_OR). The ENS_OR selects the candidate relays set and prioritizes the nodes in it based on their virtual optimal transmission distance and residual energy level. The nodes which are closer to the energy equivalent nodes and have more residual energy than the source node will be selected as the relaying nodes. In [22], the authors propose two accurate energy consumption based objective functions, which exploit the knowledge of both the frame error ratio within the physical layer, the maximum number of retransmissions in the medium access control (MAC), and the number of relays in the network layer. Based on the objective functions, the routing algorithm is designed for opportunistic routing, which employs the objective functions to prioritize the nodes in the candidate relays set. The authors in [23] exploit the geographic opportunistic routing (GOR) for Quality of Services (QoS) provisioning with both the end to end reliability and delay constraints in wireless sensor networks (WSNs); moreover, the authors also define the problem of efficient GOR for multiconstrained QoS provisioning in WSNs. Based on these conclusions, the authors propose the Efficient QoS-aware GOR protocol for the WSNs, in which the energy efficiency, latency, and the time complexity are taken into account to select the relaying nodes. The authors in [24] propose a distributed optimal community-aware opportunistic routing algorithm, in which the home-aware community model is proposed. In this algorithm, first, the routing between lots of nodes are turned to the routing between a few community homes; then the algorithm maintains an optimal candidate relays set for each home. Each home only forwards its message to the node in its optimal candidate relays set. More opportunistic routings can be found in [25], [26] and [13]; especially in [13], the author reviews the opportunistic routing algorithms in recent years and identifies and discusses the future research directions related to the opportunistic routing design, optimization, and deployment.

Recently, the fuzzy logic becomes popular in routing protocols design. In [27], the authors propose an energy-effective cross-layer routing protocol for WSNs based on fuzzy logic. In this protocol, for minimizing the energy consumption and maximizing the network lifetime, the algorithm takes the remaining battery reserve capacity, the link quality, and the transmission power of the neighbor nodes into account to select the next hop relaying nodes. In [28], for reducing the average end-to-end delay of the MANETs, the authors propose a fuzzy logic based adaptive cross-layer routing protocol for the delay-sensitive applications. In this algorithm, each node can switch between reactive routing mode and proactive routing mode based on the current node status separately. The algorithm uses the fuzzy logic controller to decide the routing mode of each node. In [29], the authors propose a routing algorithm for WSNs which can extend the network lifetime and balance the energy consumption by combining the fuzzy approach and the A-star algorithm together. The remaining battery power, the number of hops to the destination node, and the traffic loads are taken into consideration to determine the optimal routing path from the source node to the destination node. Similar in [30], to prolong the network lifetime, a fuzzy logic based energy-optimization routing protocol is proposed, in which the social welfare function is used to predict inequality of residual energy of 
neighbor nodes. The algorithm computes the degree of energy balance based on the energy inequality. The fuzzy logic system uses the degree of node closeness to the shortest path, the degree of node closeness to sink, and the degree of energy balance to achieve the routing decision. Additionally, in [31], the node density, the delay, and the number of dead nodes are used as inputs of the fuzzy logic system to select the next hop relaying node for achieving the balanced energy consumption of all the nodes with minimum delay. More related works about the fuzzy logic based routing algorithms can also be found in [32], [33], [34], and [35].

Recently, more and more researchers consider that integrate the topology control into the routing algorithm in wireless sensor network or ad hoc network is an effective way to improve the network performance. In [36], the authors proposed three alternative mathematical models for integrating topology control and routing decisions so as to prolong the lifetime of sensors. For reducing the effect of primary user (PU) activities and node mobility to the stability of links in mobile cognitive networks, in [37], the authors proposed a primary user activity prediction based joint topology control and stable routing protocol (PP-JTCSR), which can quantitatively capture channel utilization patterns of PUs. By topology control in PP-JTCSR, the most stable and shortest path can be found. In [38], the authors investigate the impact of two topology control methods for resolving the problem of void/isolated nodes appeared in geographic routing protocols, which can reduce the number of void/isolated nodes significantly. In [39], the authors have shown that joint topology control and routing assignment as an optimization problem is a NP-hard problem. For solving this problem, in this paper, the author proposed TORA (joint topology control and routing assignment) which seeks to jointly optimize topology and routing for DMesh (directional antennas in wireless mesh networks). There are also many researchers that combine the routing design with the topology control, which can be found in [40], [41], [42], and [43].

\section{PRINCIPLE OF THE SCATTER BASED FUZZY LOGIC ALGORITHM}

In this section, the principle of scatter based fuzzy logic algorithm (SBFL) will be introduced in detail.

\section{A. Problem statement}

As shown in Figure 1, the fuzzy logic system is divided into three modules: fuzzification, fuzzy logic inference, and defuzzification.

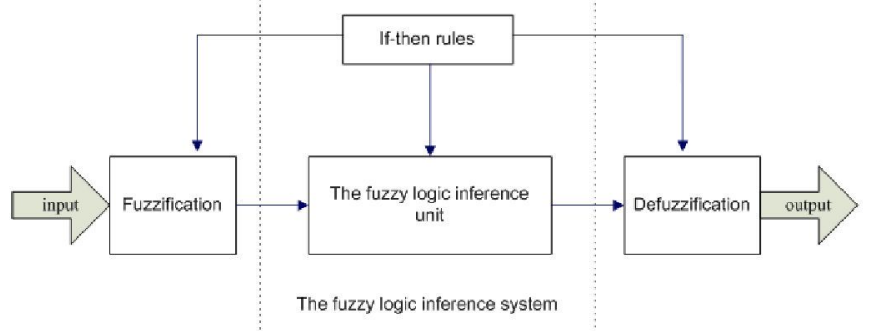

Fig. 1. The process of fuzzy logic system
In fuzzification module, the input variables are mapped to fuzzy set based on linguistic variables and membership functions. Assuming that the input universe is $U$ and the fuzzy set of universe $U$ is $A ; \mu_{A}$ is the membership function which maps universe $U$ to fuzzy set $A$. The membership function represents the membership between universe $U$ and fuzzy set $A$, i.e., $\mu_{A}: U \rightarrow A \in[0,1]$. The fuzzy set $A$ can be expressed as: $A=\left\{\left(u_{i}, \mu_{A}\left(u_{i}\right)\right) \mid u_{i} \in U\right\}$, where $u_{i}$ is the element of universe $U$. The general definition of the membership function is:

$$
\left\{\begin{array}{l}
\mu_{A}\left(u_{i}\right)=1: \quad u_{i} \text { belongs to A complete; } \\
\mu_{A}\left(u_{i}\right)=0: \quad u_{i} \text { does not belong to A complete } ; \\
0<\mu_{A}\left(u_{i}\right)<1: \quad u_{i} \text { belongs to A partly; }
\end{array}\right.
$$

The fuzzy set $A$ is the input of the fuzzy inference module which will be used to calculate the output fuzzy set $B$. The core part of the fuzzy inference system is the if-then rules (fuzzy rules). The if-then rules determine the relationship between the inputs (fuzzy set $A$ ) and outputs (fuzzy set $B$ ). The form of the fuzzy rule is generally expressed as:

$$
\text { If } A \text { (input) then } B \text { (output) }
$$

where $A$ and $B$ are the fuzzy sets of universe $X$ (input) and $Y$ (output), respectively. The membership functions are $\mu_{A}\left(x_{i}\right)$ and $\mu_{B}\left(y_{i}\right)$, where $x_{i} \in X, y_{i} \in Y$. Thus according to the fuzzy mathematics theory (Mamdani fuzzy inference algorithm), the membership function between fuzzy set $A$ and fuzzy set $B$ can be decided by:

$$
\mu_{A \rightarrow B}(x, y)=\mu_{R}(x, y) \stackrel{\Delta}{=} \mu_{A}(x) \wedge \mu_{B}(y)
$$

where $R$ represents the fuzzy relationship between universe $X$ and universe $Y$, i.e., $R=A \rightarrow B$; " $\wedge$ " is the conjunction operator in discrete mathematics. By (3), the fuzzy result can be got.

However, for taking more cross-layer performance metrics into account, the fuzzy rules will be much more complex than that shown in (2), which is a multi-inputs-single-output fuzzy system. In this scenario, the fuzzy rules will be:

$$
\text { If } A_{1}, A_{2}, A_{3}, \ldots, A_{n} \text { then } B
$$

where $A_{1}, A_{2}, A_{3}, \ldots, A_{n}$ (inputs) and $B$ (output) are the fuzzy sets of universes $U_{1}, U_{2}, U_{3}, \ldots . ., U_{n}$ (inputs) and $U$ (output), respectively. The membership functions are $\mu_{A_{1}}\left(u_{1}\right)$, $\mu_{A_{2}}\left(u_{2}\right), \quad \mu_{A_{3}}\left(u_{3}\right), \ldots \ldots, \mu_{A_{n}}\left(u_{n}\right), \quad \mu_{B}(u)$, where $u_{1} \in U_{1}$, $u_{2} \in U_{2}, u_{3} \in U_{3}, \ldots, u_{n} \in U_{n}, u \in U$. So the membership function can be expressed as:

$$
\begin{array}{r}
\mu_{\left\{A_{1}, A_{2}, A_{3}, \ldots . . A_{\left.A_{n}\right\}}\right\} B}\left(u_{1}, u_{2}, u_{3}, \ldots . ., u_{n}, u\right)=\mu_{R}\left(u_{1}, u_{2}, u_{3}, \ldots \ldots, u_{n}, u\right) \\
\stackrel{\Delta}{=} \mu_{A_{1}}\left(u_{1}\right) \wedge \mu_{A_{2}}\left(u_{2}\right) \wedge \mu_{A_{3}}\left(u_{3}\right) \cdots \wedge \mu_{A_{n}}\left(u_{n}\right) \wedge \mu_{B}(u)
\end{array}
$$

where $R=\left\{A_{1}, A_{2}, A_{3}, \ldots . ., A_{n}\right\} \rightarrow B$ is the fuzzy relationship between universe $U_{1}, U_{2}, U_{3}, \ldots ., U_{n}$ and $U$.

As shown in (5), in the multi-inputs-single-output fuzzy system, the number of the permutation and combination between the fuzzy sets are huge. The (5) illustrates that with 
the increase of the fuzzy set, the calculation complexity of the fuzzy logic system increases seriously. Moreover, if we take the number of linguistic variables into account, the calculation is too complex to be accepted. For instance, if the number of linguistic variables is $n$ and the number of fuzzy sets is $m$, then according to (5), the total number of the fuzzy rules is $n^{m}$. This means that with the increase of either the fuzzy set or the linguistic variables, the number of the fuzzy rules increases exponentially. A large number of fuzzy rules need large memory space, which is always impractical in MANETs. For solving this issue, we propose an algorithm which can isolate the effect of the number of inputs on calculation from the fuzzy inference system, i.e., the number of fuzzy rules does not increase when the number of inputs increases. This algorithm is named the scatter based balanced fuzzy logic algorithm, shorted as SBFL.

\section{B. Principle of the SBFL algorithm}

Considering the fact that during the relay node selection and prioritization, the performance metric (such as the residual energy, the ETX, the distance to the destination node, etc.) whose variation rate is large has greater effect on the routing performance than that of the metric whose variation rate is small. For instance, as the performance metrics shown in Table 1, in which the variation rate of Metric_1 is smaller than that of Metric_2. During the relaying node selection and prioritization, from the point of view of Metric_1, which node is chosen as the next hop relay node has small effect on the routing performance, since the values of Metric_1 in these three nodes are similar; however, considering the values of Metric_2, which node is chosen has greater effect on the routing performance than that of Metric_1. For evaluating the variation rate of the metrics, one of the available parameters is the variance. However, as shown in Table 1, the variance is affected seriously by the metric's value. In Table 1 , the variance of Metric_1 is larger than that of Metric_2; however, taking the metrics' values into account, the variation rate of Metric_1 is smaller than that of Metric_2 in fact. So a new parameter should be developed for reflecting the accurate variation rate of the performance metrics.

TABLE 1

METRICS AND PRIORITIES

\begin{tabular}{ccccccc}
\hline & node1 & node2 & node3 & $V$ & $r v$ & weight $^{1}$ \\
\hline Metric_1 & 1001 & 1002 & 1003 & 0.667 & $6.64 \times 10^{-6}$ & 0.0523 \\
\hline $\begin{array}{c}\text { Order of } \\
\text { Metric_1 }\end{array}$ & 1 & 2 & 3 & & & \\
Metric_2 & 0.5 & 0.8 & 0.1 & 0.0822 & 0.377 & 0.753 \\
$\begin{array}{c}\text { Order of } \\
\text { Metric_2 }\end{array}$ & 2 & 3 & 1 & & & \\
$\begin{array}{c}\text { Metric_3 } \\
\text { Order of }\end{array}$ & 1000 & 2000 & 3000 & 666667 & 0.167 & 0.333 \\
$\begin{array}{c}\text { Metric_3 } \\
\text { Node } \\
\text { utility }\end{array}$ & 1 & 2 & 3 & & & \\
\hline
\end{tabular}

${ }^{1}$ this weight is calculated by the traditional fuzzy logic algorithm, in which the number of linguistic variables is 3 [29];

${ }^{2}$ this utility is calculated by (12).

In SBFL algorithm, assuming that there are $n$ input universes, which is $U=\left[U_{1}, U_{2}, U_{3}, \ldots \ldots, U_{n}\right]$; for each universe, there are $m$ elements, i.e. $U_{i}=\left[U_{i}^{1}, U_{i}^{2}, U_{i}^{3}, \ldots \ldots, U_{i}^{m}\right]^{T}$; therefore,

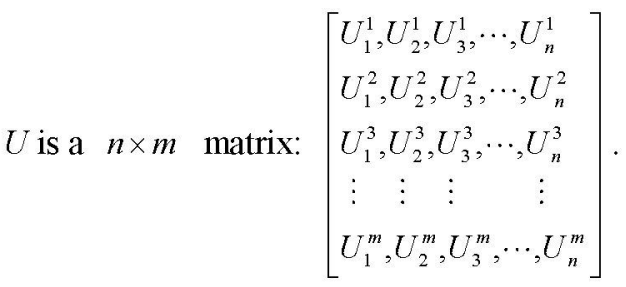

For reflecting the variation rate of the metric more accurately, we propose the relative variance $(r v)$ which takes the average value of the metrics into account to evaluate the variation rate of the metric, defined as:

$$
D_{i}=\frac{1}{m} \sum_{j=1}^{m}\left(\frac{U_{i}^{j}-\bar{U}_{i}}{\bar{U}_{i}}\right)^{2}, i \in[1, n]
$$

where $U_{i}^{j}$ means the $j$ th element of $U_{i} ; \bar{U}_{i}$ is the mean value of the parameters in $U_{i}$ and can be calculated as:

$$
\bar{U}_{i}=\frac{1}{m} \sum_{j=1}^{m} U_{i}^{j}, i \in[1, n]
$$

In (6), the variation rate is not affected by the metric's value, which can be found in Table 1. In Table 1, even the variance of Metric 1 is larger than that of Metric_2, the $r v$ of Metric_1 is much smaller than that of Metric_2; so the relative variance can reflect the variation rate of the metric more accurately than variance.

In SBFL, the input universes $U_{1}, U_{2}, \ldots, U_{n}$ are replaced by the relative variance $D$ as the input of the fuzzy inference system. The fuzzy set $A_{D}$ can be expressed as:

$$
\begin{aligned}
A_{D} & =\left\{\left(d, d_{A}\left(d_{i}\right)\right) \mid d_{i} \in D\right\} \\
& =\left[d_{A}\left(d_{1}\right), d_{A}\left(d_{2}\right), d_{A}\left(d_{3}\right), \ldots . ., d_{A}\left(d_{n}\right)\right]
\end{aligned}
$$

where $d_{A}\left(d_{i}\right)$ is the membership function between scatter $D$ and fuzzy set $A_{D}$. Since the scatter $D$ is a $1 \times n$ matrix, so the fuzzy rules shown in (5) can be rewritten as: If $A_{D}$ then $B$. Therefore, the membership function between $A_{D}$ and $B$ will be:

$$
\mu_{D \rightarrow B}(z, y)=\mu_{R}(z, y) \stackrel{\Delta}{=} \mu_{D}(z) \wedge \mu_{B}(y)
$$

As shown above, the membership function in (9) is much simpler than that in (5). By using the concept of relative variance, the number of the fuzzy sets has been reduced from $n$ to 1 , so the calculation complexity is reduced. Moreover, the number of fuzzy rules in SBFL does not increase when the number of fuzzy set and the linguistic variables increase. Additionally, as shown in Figure 2, the complexity caused by the increase of the number of inputs has been isolated from the fuzzy inference system successfully. This means no matter how many different universes are used, the number of fuzzy logic input is only one by using SBFL, which means that the computation complexity is low. 

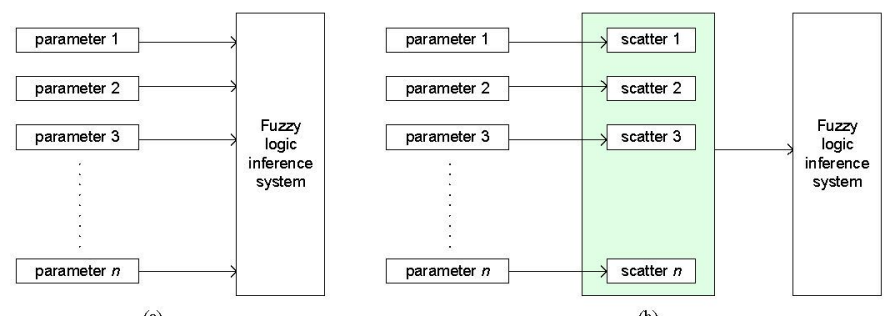

(a)

Fig. 2. (a) The principle of the traditional fuzzy logic system; (b) The principle of SBFL algorithm.

The outputs of SBFL are the weights of the cross-layer metrics:

$$
\omega=\left[\omega_{1}, \omega_{2}, \omega_{3}, \omega_{4} \ldots \ldots, \omega_{m}\right]
$$

In SBFL, the cross-layer metrics whose $r v$ are high have great effect on the relay node selection, vice versa; therefore, the weights of the metrics are large when their $r v$ are large; otherwise, the weights are small. Moreover, as the number of the linguistic variables in SBFL can be set as large as possible, so according to [14], the number of the linguistic variables is set to 7 in this paper (the number of linguistic variables can be any positive integer; the more linguistic variables, the more accuracy of the result is; however, the computation complexity increases slightly). The fuzzy linguistic variables and the fuzzy rules are shown in Table 2.

TABLE 2

FUZZY LOGIC RULES

\begin{tabular}{l|l}
\hline IF Input $(D)$ & THEN Output $(\omega)$ \\
\hline very small & very small \\
medium small & medium small \\
small & small \\
medium & medium \\
large & large \\
medium large & medium large \\
very large & very large \\
\hline
\end{tabular}

When the weight of each cross-layer metric has been got, the $\omega$ and $U$ will be used to calculate the utilities of nodes in CRS:

$$
\begin{aligned}
\psi=\omega \times U & =\left[\psi_{1}, \psi_{2}, \psi_{3}, \psi_{4}, \ldots \ldots, \psi_{n}\right] \\
& =\left[\sum_{j=1}^{m} \omega_{j} \cdot U_{1}^{j}, \sum_{j=1}^{m} \omega_{j} \cdot U_{2}^{j}, \ldots, \sum_{j=1}^{m} \omega_{j} \cdot U_{n}^{j}\right]
\end{aligned}
$$

However, the important issue should be paid attention in SBFL is that the order-of-magnitude of the metrics are different, which have great effect on the algorithm. If we use the values of these metrics directly in SBFL algorithm, there will have mistakes. For instance, considering the metrics shown in Table 1, the values in Metric_3 is much larger than that in Metric_2 and Metric_1. If the SBFL algorithm is applied directly, the utilities of node1, node2, and node 3 are 385.73, 719, and 1051.54, respectively. As a result, the priorities of these nodes are: node $3 \rightarrow$ node $2 \rightarrow$ node 1 ; however, these node utilities are decided mainly by the value of Metric_3. The Metric_3 will cover up the effect of Metric_1 and Metric_2 on the relay node selection. The reason is that the metrics in Table 2 are not in the same order-of-magnitude; therefore, the parameters whose order-of-magnitudes are larger than others have great effect on the SBFL algorithm.

In this paper, for solving this issue, we introduce the metric priority into the node utility calculation. The metric priority can reflect the relationship of these metrics. For instance, as the metrics in Table 1, the priorities of each metrics are given. The priority of Metric_i is denoted as $R_{\text {Merric } i}$. So the (11) can be rewritten as:

$$
\begin{aligned}
\psi & =\omega \times R=\left[\psi_{1}, \psi_{2}, \psi_{3}, \psi_{4}, \ldots \ldots, \psi_{n}\right] \\
& =\left[\sum_{j=1}^{m} \omega_{j} \cdot R_{\text {Metric_1 }}^{j}, \sum_{j=1}^{m} \omega_{j} \cdot R_{\text {Metric_2 }}^{j}, \ldots, \sum_{j=1}^{m} \omega_{j} \cdot R_{\text {Metric } \_n}^{j}\right]
\end{aligned}
$$

According to (12), the utilities of these three nodes are shown in Table 1. By using (12), the node priorities will be: node $2 \rightarrow$ node $3 \rightarrow$ nodel, which is more reasonable than that of the node utilities that calculated by (11). As shown in Table 1, the orders of the node utilities are decided by these three performance metrics jointly; the order of the node utilities is different with the order of any performance metrics.

A simple example can be found as follows. In this example, we will evaluate the effectiveness of SBFL algorithm. The parameters are shown in Table 3. There are five nodes and three metrics for each node.

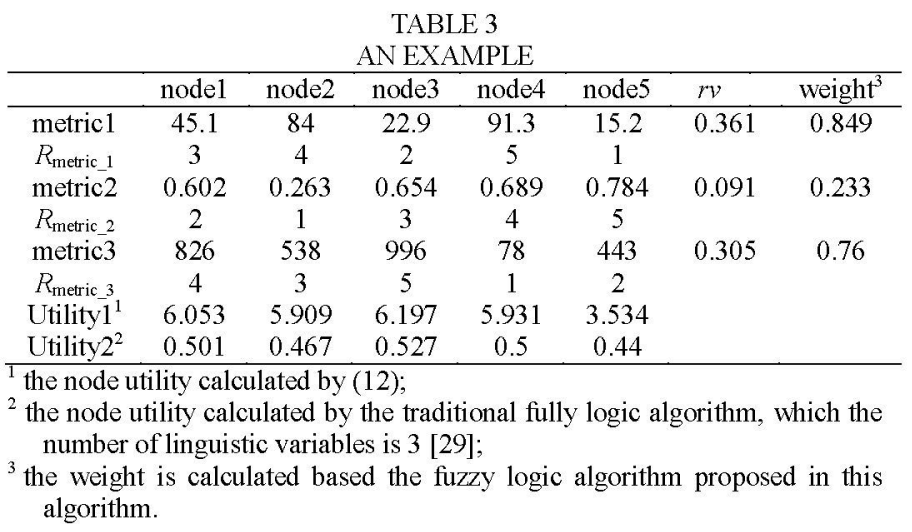

Table 3 shows the performance of the traditional fuzzy logic algorithm and SBFL algorithm on determining the nodes' priorities, respectively. Table 3 demonstrates that based on SBFL algorithm, the utilities of node1, node2, and node4 are similar, which are larger than node5; the utility of node 3 is the largest in these five nodes. Therefore, the priorities of these nodes are: node $3 \rightarrow$ node $1 \rightarrow$ node $4 \rightarrow$ node $2 \rightarrow$ node 5 . The priorities of the nodes that decided by the traditional fuzzy logic algorithm are also presented in Table 3, which are: node $3 \rightarrow$ node $1 \rightarrow$ node $4 \rightarrow$ node $2 \rightarrow$ node 5 ; this is the same as the node utilities that calculated by (12). This demonstrates that the approach shown in (12) is effective on determining the relay priorities of the relay nodes.

Another disadvantage of the traditional fuzzy logic algorithm is the calculation complexity. In this simulation, to the traditional fuzzy logic algorithm, the number of the performance metric is 3 in each node and the number of the linguistic variable is 3 , so the number of fuzzy rules is 27 ; however, in the SBFL algorithm, this number is only 7, which is equal to the number of the linguistic variables. This can be found in Figure 3. In Figure 3(a), with the increase of the fuzzy sets, the number of the fuzzy rules in traditional fuzzy 
logic algorithm increases exponentially; however, this number will keep constant in SBFL algorithm. The similar result can be found in Figure 3(b). In Figure 3(b), with the increase of the linguistic variables, the fuzzy rules in the traditional fuzzy logic system increase exponentially like that in Figure 3(a); however, in SBFL algorithm, the increase is slight and equals to the number of the linguistic variables. Figure 3 demonstrates that the SBFL algorithm has great advantages on dealing with the multi-inputs issues.

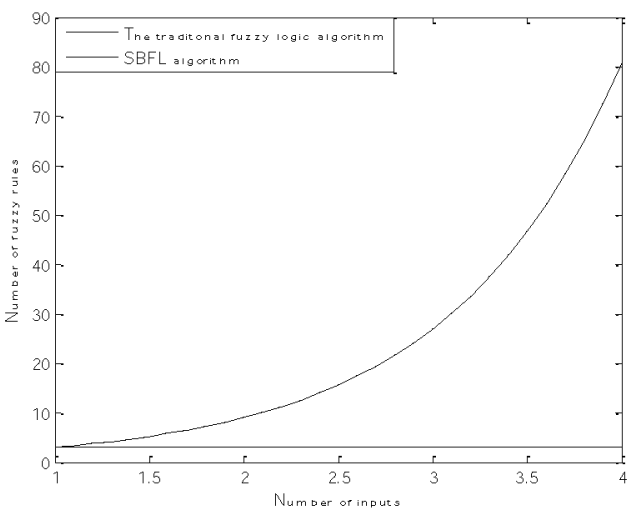

(a)

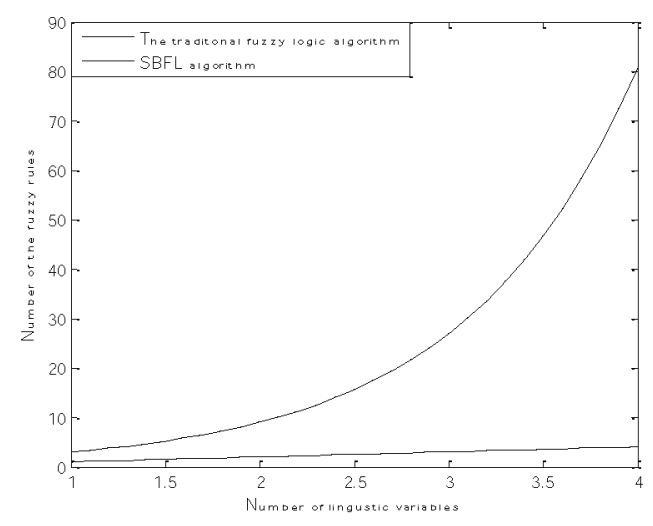

(b)

Fig. 3. The number of rules: (a) the number of linguistic variables is fixed; (b) the number of the fuzzy set is fixed.

\section{CROSS-LAYER BALANCED AND RELIABLE OPPORTUNISTIC ROUTING ALGORITHM}

Based on the SBFL algorithm that proposed in Section 3, we propose the cross-layer and reliable opportunistic routing algorithm (CBRT) for MANETs in this section. Considering the node mobility in MANETs, the topology control algorithm and the link lifetime prediction algorithm which takes both the moving speed and the moving direction into account are developed in this section.

\section{A. Link lifetime prediction algorithm}

In MANETs, due to the node mobility, the network topology changes frequently, so the route easy to be broken and cannot last a long time [44]. The link lifetime is affected by the node mobility seriously. The node mobility includes the moving speed and moving direction. Both these two parameters can affect the link lifetime. However, in the previous works, the effect of the moving direction on the link lifetime has not been fully investigated. In this section, this issue will be investigated in detail.

The link lifetime prediction is difficult in MANETs because the nodes can move freely [44]. During the calculation, we assume that the link connections are unstable and can only last a short period of time [44]; so we can assume reasonably and simply that the nodes move at a constant speed and do not change their moving direction in such a short period [44][56-59]. Moreover, in CBRT, each node equips a GPS device [44], which can be used to obtain the node's coordinate and the moving speed and direction [44][56-59].

The node acquires other nodes' location by periodical probe packet exchanging. In this algorithm, only the neighbors whose distances to the destination node are smaller than that of the source node can be considered as the candidate relay nodes. As shown in Figure 4, when the nodes located in the red area (which is defined as the survival area; the survival area means that in this area, the link connection can be guaranteed), the link lifetimes are larger than 0 . The value of the link lifetime relates to the source node $s$, the relaying node $r$, and the destination node $d$. Due to the mobility of the source node $s$ and the destination node $d$, the survival area changes continuously. In addition, considering the mobility of the relaying node $r$, the communication link will be broken when the node $r$ moves out of the survival area. Therefore, the relative velocity of node $r$ relative to node $s$ and node $d$ need to be calculated. The calculation of the relative velocity is shown in Figure 5.

The proposed link lifetime prediction algorithm is different with the previous works on the following aspects: 1) in the previous works, the link lifetime prediction need extra sample packets, such as, in [56], three sample packets are needed during the prediction, in [44], two sample packets are needed; however, in CBRT, no extra sample packet is needed; 2) in the previous works, the prediction area is the whole coverage area of the node and the coverage area only relates to node $s$ and does not change relative to node $s$, i.e., the cycle shown in Fig. 4 ; however, in this paper, the prediction area is the survival area, which relates to both the node $s$ and node $d$, and changes continuously relative these two nodes; so it puts more challenges than the previous works; 3 ) in the previous works, the moving direction of the node is not taken into account during the link lifetime prediction.

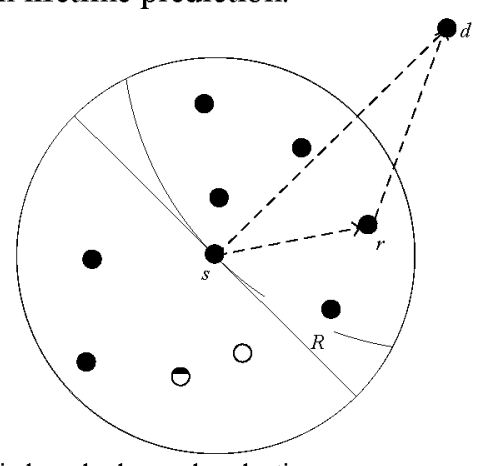

Fig. 4. Geographic based relay node selection 

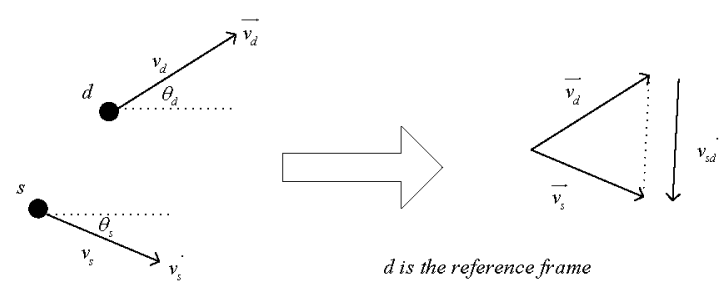

dis the reference frame

Fig. 5. The principle of the velocity vector operation

In Figure 5, the moving velocities of node $s$, node $r$, and node $d$ are $\vec{v}_{s}$ (the moving speed is $v_{s}$ and the moving direction is $\theta_{s}$ ), $\vec{v}_{r}$ (the moving speed is $v_{r}$ and the moving direction is $\theta_{r}$ ), and $\overrightarrow{v_{d}}$ (the moving speed is $v_{d}$ and the moving direction is $\theta_{d}$ ), respectively. If the destination node $d$ is chosen as the reference frame, then the relative velocity of source node $s$ relative to node $d$ is given by: $\overrightarrow{v_{s d}}=\overrightarrow{v_{s}}-\overrightarrow{v_{d}}$. According to the vector synthesis theory, the relative moving speed and moving direction of $\overrightarrow{v_{s d}}$ can be given by: $v_{s d}=\sqrt{v_{x s d}^{2}+v_{y s d}^{2}}, \theta_{s d}=\arctan \left(v_{x s d} / v_{y s d}\right)$, $v_{x s d}=v_{s} \cos \theta_{s}+v_{d} \cos \theta_{d}$, and $v_{y s d}=v_{s} \sin \theta_{s}+v_{d} \sin \theta_{d}$; where $v_{x s d}$ is the moving speed of $v_{s d}$ in $x$-axis and $v_{y s d}$ is the moving speed of $v_{s d}$ in $y$-axis. Therefore, the relative velocity of relay node $r$ relatives to $\overrightarrow{v_{s d}}$ can be calculated as: $\overrightarrow{v_{s c i}}=\overrightarrow{v_{r}}-\overrightarrow{v_{s d}}$. Similar to that shown above, the relative moving speed and moving direction of $\overrightarrow{v_{s d r}}$ can be got from: $v_{s d r}=\sqrt{v_{x s d r}^{2}+v_{y s d r}^{2}} \quad, \quad \theta_{s d r}=\arctan \left(v_{x s d r} / v_{y s d r}\right)$ $v_{x s d r}=v_{r} \cos \theta_{r}+v_{s d} \cos \theta_{s d}$, and $v_{s s d r}=v_{r} \sin \theta_{r}+v_{s d} \sin \theta_{s d}$, where $v_{x s d r}$ is the speed of $v_{s d r}$ in $x$-axis and $v_{y s d r}$ is the speed of $v_{s d r}$ in y-axis.

As shown in Figure 6, node $r$ moves with velocity $\overrightarrow{v_{s d r}}$. Assuming that the node $r$ will move out of the survival area at time $t$, according to Figure 6, there are two different scenarios about this issue: (1) the relaying node moves toward to the destination node; (2) the relaying node moves far away from the destination node. For solving this problem, we need to calculate the relative velocity angle for node $r$.

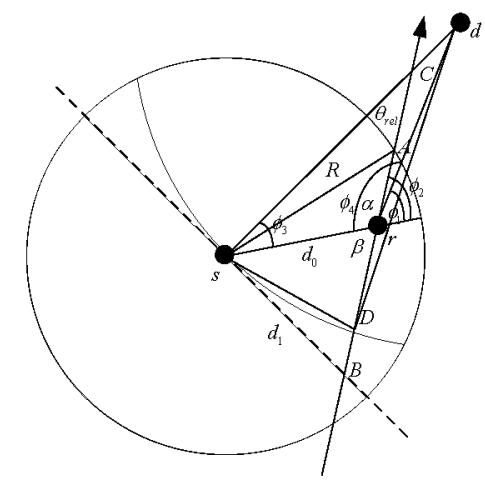

Fig. 6. The principle of the residual link lifetime calculation

As shown in Figure 6, assuming the coordinate of node $s$ is $\left(x_{s}, y_{s}\right)$ and the coordinate of node $d$ is $\left(x_{d}, y_{d}\right)$, then the angle of $\overline{s d}$ relative to the $x$-axis can be got from: $\theta_{\overline{s d x}}=\arctan \left(\frac{y_{s}-y_{d}}{x_{s}-x_{d}}\right)$. According to $\theta_{s d r}=\arctan \left(v_{x s d i r} / v_{y s d r}\right)$ and the triangle geometry theory, the velocity angle of node $r$ relative to $\overline{s d}$ is: $\theta_{r e l}=\theta_{s d r}-\theta_{s d x}$.

Therefore, if $\theta_{r e l} \in[0, \pi / 2] \cup[3 \pi / 2,2 \pi]$, the node $r$ moves close to the destination node in $x$-axis or $y$-axis; otherwise, when $\theta_{r e l} \in[\pi / 2,3 \pi / 2]$, the node $r$ moves far away from the destination node in both $x$-axis and $y$-axis. So the link lifetime prediction should be divided into two different scenarios.

1. When $\theta_{r e l} \in[0, \pi / 2] \cup[3 \pi / 2,2 \pi]$

In this scenario, the relay node $r$ moves toward to the destination node $d$ in $x$-axis or $y$-axis. According to Figure 5 and the law of cosines, in triangle $s r d$ we can get the function as follows:

$$
d_{d r}^{2}=d_{0}^{2}+d_{d s}^{2}-2 d_{0} d_{d s} \cos \phi_{3}
$$

where $d_{d r}$ is the distance between relay node $r$ and destination node $d ; d_{0}$ is the distance between source node $s$ and relay node $r$ at the time $t_{0} ; d_{d s}$ is the distance between source node $s$ and destination node $d$. Since the values of $d_{d r}$, $d_{0}$, and $d_{d s}$ can be calculated based on the coordinates of these nodes, so the value of $\phi_{3}$ can be got from (13). Therefore, $\phi_{2}$ can be calculated as: $\phi_{2}=\pi-\phi_{3}$.

Additionally, in triangle $s r C, \theta_{r e l}$ and $\phi_{3}$ are all known, so the angle $\alpha$ can be calculated: $\alpha=\pi-\phi_{3}-\theta_{\text {rel }}$. In triangle $s r A$, according to the law of cosines, we can get:

$$
R^{2}=d_{0}^{2}+\left[v_{s d r}\left(t-t_{0}\right)\right]^{2}-2 d_{0} v_{s d r}\left(t-t_{0}\right) \cos \alpha
$$

where $R$ is the transmission range of source node $s$. Therefore, the $t$ can be calculated by (14).

Thus in this scenario, the residual link lifetime is:

$$
T_{r}=t-t_{0}
$$

2. When $\theta_{r e l} \in[\pi / 2,3 \pi / 2]$

When $\theta_{r e l} \in[\pi / 2,3 \pi / 2]$, the link lifetime prediction is similar to that when $\theta_{r e l} \in[0, \pi / 2] \cup[3 \pi / 2,2 \pi]$. The distance between the destination node $d$ and relay node $r$ at time $t_{0}$ is $d_{d r}$ and the length of line $d D$ is equal to $d_{d s}$. Moreover, in triangle $d r D$, the $\angle d r D$ can be calculated as:

$$
\angle d r D=\phi_{5}=\pi-\left(\phi_{2}-\phi_{1}\right)
$$

Additionally, in triangle $s r d$, according to the law of cosines we can get:

$$
\begin{gathered}
d_{d s}^{2}=d_{0}^{2}+d_{d r}^{2}-2 d_{0} d_{d r} \cos \phi_{4} \\
\phi_{1}=\pi-\phi_{4}
\end{gathered}
$$

In (16), the $\phi_{2}$ can be calculated by $\phi_{2}=\pi-\phi_{3}$ and $\phi_{1}$ can be calculated by (17) and (18), so in triangle $d r D$, we have:

$$
d_{d s}^{2}=d_{d r}^{2}+\left[v_{s c t r}\left(t-t_{0}\right)\right]^{2}-2 d_{d r} v_{s d r}\left(t-t_{0}\right) \cos \phi_{5}
$$

In (19), the $t$ can be calculated. So the residual link lifetime in this scenario can be calculated by (15).

\section{B. Packet delivery ratio based opportunistic topology control algorithm}


In this section, we propose the packet delivery ratio based opportunistic topology control algorithm (OTC). The OTC is inspired by the humoral regulation, in which the feasible solution is a range rather than a single value. In OTC, the transmission power is adjusted dynamically to maintain the stability of the packet delivery ratio; moreover, it can reduce the topology control cost by dividing the nodes into different categories.

Before proposing the topology control algorithm, we first define the necessary definitions which will be used in this section.

Definition 1: The relay node degree (RND) is defined as the number of neighbors whose distances to the destination node are smaller than that of the source node, i.e., the number of nodes in the survival area (shown in Figure 4).

Definition 2: The transmission power adjustment ratio is defined as the ratio of the number of nodes which adjust their transmission power to the number of nodes in the whole network, which can be expressed as:

$$
C_{O T C}=\frac{\text { nodes adjust the transmission power }}{\text { total nodes in the network }} .
$$

The transmission power adjustment ratio is the smaller the better; since the small transmission power adjustment ratio means that there is small part of nodes in the network need to change their transmission power, which also means less control cost of the network.

In opportunistic routing algorithm, the packet delivery ratio between source node and CRS can be calculated as [20]:

$$
P T P=1-\left(1-p_{i}\right)^{n}
$$

where $p_{i}$ is the packet delivery ratio between sender and one neighbor node, and can be determined by periodically beacon message exchanging between sender and its neighbors [45][46][53]; $n$ is the RND of the CRS. According to (20), to different RND of the CRS, the packet delivery ratio varies greatly, especially in MANETs. For getting stable packet delivery ratio, the RND should keep constant in CRS. However, due to the high mobility of MANETs, the network topology changes frequently; so if each node maintains constant RND in CRS, the transmission power needs to be adjusted frequently and the transmission power adjustment ratio is high. The high adjustment ratio makes the communication channel more and more congested. Therefore, for reducing the adjustment ratio, the nodes are divided into different categories in OTC according to their packet delivery ratio. Whether the transmission ranges need to be adjusted or not will be decided by which categories that the nodes belong to. The definitions of different categories are shown in Definition 3.

Definition 3. When the packet delivery ratio of the node $(P T P)$ is in the region $\left(P_{1}, P_{2}\right)$; then the node is healthy; otherwise, the node is unhealthy.

The definition is shown in Figure 7 and expressed in (34).

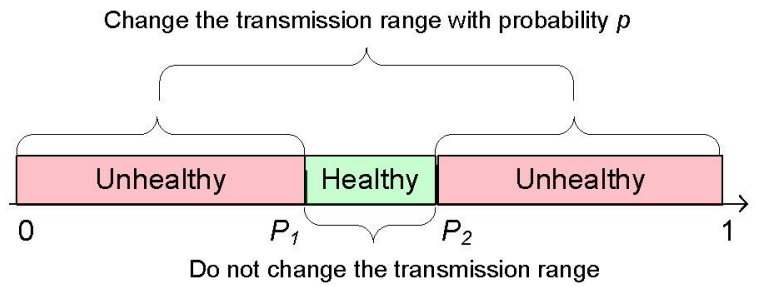

Fig. 7. The definition of the different categories

$$
\left\{\begin{array}{l}
R_{H} \in\left(P T P \mid P T P \in\left[P_{1}, P_{2}\right]\right) \\
R_{U H} \in\left(P T P \mid P T P \in\left[0, P_{1}\right) \cup\left(P_{2}, 1\right]\right)
\end{array}\right.
$$

where $P_{1}$ and $P_{2}$ are the boundary values of different categories; $R_{H}$ means the healthy region; $R_{U H}$ means the unhealthy region. To the specific packet delivery ratio, the needed RND of the CRS can be calculated by (20). So the (21) can also be expressed by RND, which is:

$$
\left\{\begin{array}{l}
R_{H} \in\left(n \mid n \in\left[n_{1}, n_{2}\right]\right) \\
R_{U H} \in\left(n \mid n \in\left[0, n_{1}\right) \cup\left(n_{2}, N\right]\right)
\end{array}\right.
$$

where $n$ is RND, $N$ is the node number in the network.

The source node calculates the RND in the candidate relays set and adjusts the transmission power according to the value of RND. For reducing the transmission power adjustment ratio, in OTC, the transmission power adjustment probabilities are different in different regions. In health region, this probability is 0 ; however, in the unhealthy region, this probability varies from 0 to 1 based on the value of RND, which can be expressed as:

$$
\left\{\begin{array}{l}
p_{h}=0, n \in\left[n_{1}, n_{2}\right] \\
p_{u}=(0,1], n \in\left[0, n_{1}\right) \cap\left(n_{2}, N\right]
\end{array}\right.
$$

where $p_{h}$ is the transmission power adjustment probability of healthy region; $p_{u}$ is the probability of unhealthy region.

In the unhealthy region, the adjustment probability varies and is decided by the deviation of the RND in the unhealthy region to the boundary values. When the RND is far from the healthy region, the adjustment probability should be high to guarantee the reliability and packet delivery ratio, vice versa. So the adjustment probability can be calculated as:

$$
p_{s i}=\left\{\begin{array}{l}
\left(n_{1}-n_{i}\right) / n_{1}, n_{i} \in\left[0, n_{1}\right) \\
\left(n_{i}-n_{2}\right) /\left(N-n_{2}\right), n_{i} \in\left(n_{2}, N\right]
\end{array}\right.
$$

where $n_{i}$ is the RND of node $i ; p_{s i}$ is the transmission power adjustment probability of node $i$ and $0 \leq p_{s i} \leq 1$.

As shown in [47], the probability that the node number is $n$ in the coverage area of the node is Poisson distribution; therefore, the probability that there are $n$ nodes in the survival area is:

$$
P(n)=\frac{(\rho \Delta)^{n}}{n !} e^{-\rho \Delta}
$$

where $\Delta$ is the survival area that shown in Figure $4 ; \rho$ is 
the node density of the network; $n$ is the RND in the CRS.

According to (25), the probability that the RND is in the region $\left[n_{1}, n_{2}\right]$ can be calculated as:

$$
P\left(n_{1} \leq n \leq n_{2}\right)=\sum_{n=n_{1}}^{n_{2}} \frac{(\rho \Delta)^{n}}{n !} e^{-\rho \Delta}
$$

When $\left.P^{\prime}\left(n_{1} \leq n \leq n_{2}\right)\right|_{\Delta}=0$, the (26) will get the maximum value. This means the probability that there are $n$ nodes in the survival area $\Delta$ is the highest. Moreover:

$$
\left.P^{\prime}\left(n_{1} \leq n \leq n_{2}\right)\right|_{\Delta}=\sum_{n=n_{1}}^{n_{2}} \frac{1}{n !}(\rho \Delta)^{n-1}\left(n \rho e^{-\rho \Delta}-(\rho \Delta) \rho e^{-\rho \Delta}\right)
$$

when $\left.P^{\prime}\left(n_{1} \leq n \leq n_{2}\right)\right|_{\Delta}=0$, we can get the optimal survival area $\Delta^{*}$.

In Figure 6, the transmission range of node $s$ is $r$ and the distance between node $s$ and node $d$ is $d$. So the line $B d$ is also equal to $d$. Then the triangle $\mathrm{s} B d$ is an isosceles triangle. Therefore, the angle of $\theta_{B s d}$ can be calculated as:

$$
\theta_{B s d}=\arccos (r /(2 d))
$$

Then the optimal value of the transmission range can be calculated as:

$$
\Delta^{*}=r^{2} \cdot \arccos (r /(2 d))
$$

where $r^{2} \cdot \arccos (r /(2 d))$ is the area of the survival area.

Based on (28) and (29), we can get the optimal transmission range $r^{*}$ for the source node. This transmission range has the highest probability to guarantee that the RND in CRS is in the region $\left[n_{1}, n_{2}\right]$. Therefore, when the node is in the unhealthy region, the node transmission ranges will be adjusted to $r^{*}$.

Therefore, according to (24) and Definition 2, we can calculate the transmission power adjustment ratio of OTC as follows:

$$
C_{\text {OTC }}=P_{u}+\frac{1}{N} \sum_{i=1}^{N P_{s}} p_{s i}
$$

where $P_{u}$ means the probability that the node is in the unhealthy region; $p_{s i}$ is the transmission power adjustment probability of node $i ; N$ is the node number in the network. According to (25) and (26), the $P_{u}$ can be calculated as:

$$
\begin{aligned}
P_{u} & =P\left[\left(0 \leq n<n_{1}\right) \cup\left(n_{2}<n \leq N\right)\right] \\
& =\sum_{n=0}^{n_{1}-1} \frac{\left(\rho \Delta^{*}\right)^{n}}{n !} e^{-\rho \Delta^{*}}+\sum_{n=n_{2}+1}^{N} \frac{\left(\rho \Delta^{*}\right)^{n}}{n !} e^{-\rho \Delta^{*}}
\end{aligned}
$$

There are two application-specific parameters in OTC, which are the boundary values of the healthy region: $P_{1}$ and $P_{2}$ Since different applications have different network parameters and QoS requirements, so these two parameters are not fixed; they are different with different applications. For instance, if the application requires that the packet delivery ratio should larger than 0.9 , then the healthy region can be decided as $[0.9,0.99]$, since the probability can not equal to 1 ; then the RND can be calculated based on these two probabilities and (20).

\section{Cross-layer and reliable opportunistic routing algorithm}

Based on the link lifetime prediction algorithm and the OTC algorithm, we propose the cross-layer balanced and reliable opportunistic routing algorithm for MANETs in this section. The data packet transmission in opportunistic routing is store-and-forward.

In CBRT, each node acquires the locations of other nodes in the network through periodic probe packet exchanging. Only the locations of other nodes are maintained periodically by each node. Once the source node wants to send data packet to the destination node, first, it will broadcast the RREQ (Route Request) to its neighbors. The neighbors are the nodes who have one-hop bi-directional communication links with the source node. The RREQ includes the node IDs of the source node and the destination node. The neighbors who receive this message will calculate the distances to the destination node. The neighbor nodes whose distances to the destination node are larger than the distance between the source node and destination node will drop the RREQ packet directly. Only the neighbor nodes whose distances to the destination node are smaller than the source node will reply RREP (Route Request Response) message to the source node.

In CBRT, we take several performance metrics into account during the routing decision. For these performance metrics, only the nodes' location needed to be maintained by each node periodically. For the rest of performance metrics, the source node acquires them only when there is a routing request. That means when the source node begins a routing discovery process and sends RREQ to the neighbor nodes, the neighbor nodes whose distances to the destination node are smaller than the source node will reply the RREP to the source node; in this RREP packet, the concerned cross-layer performance metrics (including the residual energy, moving speed and direction, etc.) are included, i.e., piggyback information of RREQ and RREP packets during the routing discovery [44]. If there has no routing request, the source node does not need to maintain these performance metrics, except the other nodes' location.

When the source node receives the RREP packet, it will calculate the RND in CRS and decide which region that it belongs to according to the value of RND. The source node adjusts its transmission power based on OTC algorithm. After the transmission power adjustment, the source node will update the CRS. If the optimal transmission power is smaller than the previous one, then the source node deletes the nodes whose distances to the source node are larger than the transmission range from the CRS. If the transmission power is larger than the previous one, the source node needs to broadcast RREQ packet again. Only the nodes which are not in the CRS and the distances to the destination node are smaller than the source node reply RREP message to the source node. After the CRS updating, the source node extracts the cross-layer parameters from the RREP packets. According to (6), the source node calculates the scatter $D_{i}$ of each cross-layer parameters. These scatters $D_{i}$ are the inputs of the fuzzy logic inference system. The source node relays the data packet to the nodes in CRS. The transmission priorities of the relaying nodes in CRS are decided by the utilities that 
calculated by (12). When the relaying nodes receive the data packet, they will repeat the transmission process that introduced above till the data packet is received by the destination node.

The process of the cross-layer balanced and reliable opportunistic routing algorithm is shown as follows:

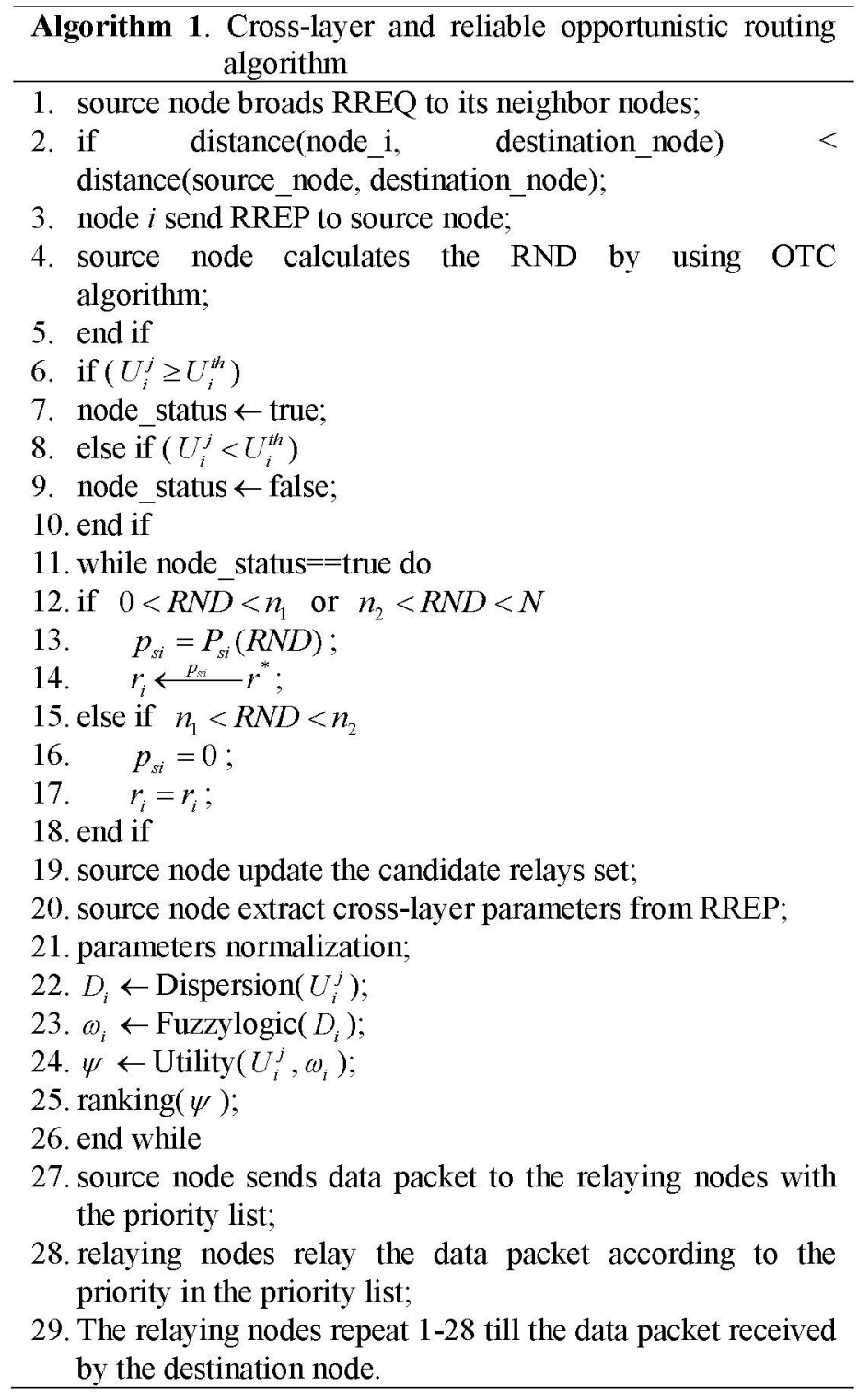

\section{SIMULATION AND DISCUSSION}

In this section, the performance of CBRT algorithm will be evaluated and compared with the ExOR algorithm. The effectiveness of SBFL algorithm has been demonstrated in Section 3; so in the first part of this section, the performance of the packet delivery ratio based topology control algorithm is presented; in the second part of this section, the performance of CBRT and ExOR will be compared in detail. The simulator is NS-2 in this simulation.

A. Performance of packet delivery ratio based opportunistic topology control algorithm (OTC)

In this section, the performance of the packet delivery ratio based opportunistic topology control algorithm will be evaluated.

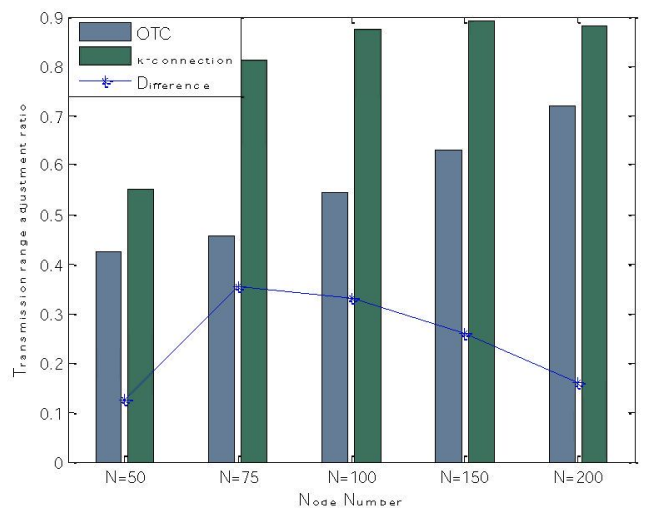

Fig. 8. The transmission power adjustment ration of OTC and $k$-connection algorithm.

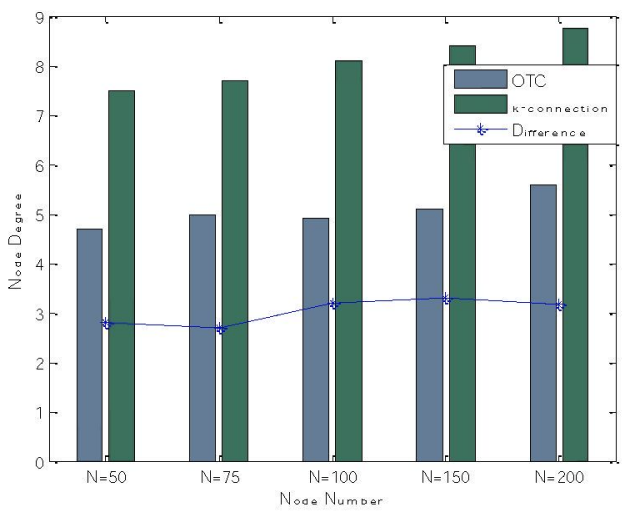

Fig. 9. The node degree of OTC and $k$-connection algorithm.

In this simulation, we compare the OTC with the traditional $k$-connection algorithm which is the most popular topology control algorithm in wireless sensor and ad hoc networks $[48][49][50]$. In this simulation, the boundary values of the different regions are the same as that shown in Section 4; the value of $k$ in the $k$-connection algorithm is equal to 5 . The simulation results can be found in Figure 8 and Figure 9. The parameters of the simulation are: deployment area: $1000 \mathrm{~m} \times 1000 \mathrm{~m}$; initial node transmission range: $100 \mathrm{~m}$; traffic type: CBR (constant bit rate); CBR rate: 512 Bytes $\times 6$ per second [44][53]; initial energy: 5J; high transmission power: $0.8 \mathrm{~W}$; low transmission power: $0.1 \mathrm{~W}$; node number $50-200$; average moving speed: $0.2 \mathrm{~m} / \mathrm{s}$; receiving power: $0.05 \mathrm{~W}$.

From Figure 8, we can conclude that the transmission power adjustment ratio of OTC is smaller than that of the $k$-connection algorithm. With the increase of the node number, both the adjustment ratios of OTC and k-connection increase. Additionally, when the number of node increases, the difference of the adjustment ratios between OTC algorithm and $k$-connection algorithm decreases. In Figure 9, the node degrees of $k$-connection algorithm vary around 5 , which is consistent with the value that we set in this simulation. In OTC algorithm, the node degree varies from 7 to 9 which are the boundary values that calculated in Section 4 . The results in Figure 9 illustrates that the OTC algorithm is effective on controlling the network topology. 


\section{B. Performance of the cross-layer and reliable opportunistic routing algorithm (CBRT)}

As demonstrated in Section 3, since the SBFL algorithm can handle more cross-layer parameters than the traditional algorithms without increasing the computation complexity, so in this simulation, the residual energy of the relaying node, the ETX of the communication link, the packet queue length of the relaying node, the transmission delay of the relaying node, the distance to the destination node, the moving speed, and the moving direction will be taken into account to choose and prioritize the relay nodes for the opportunistic routing. All these metrics are local metrics and can be calculated or obtained as follows.

1. The residual energy. The residual energy is a local metric of the node; the nodes can acquire their energy status by the detection circuit in node [27][29][51][52];

2. The ETX of the link. The link's ETX is defined as the expected number of transmissions needed to send a packet to the relay node [53]. It can be calculated as: $\frac{1}{\left(1-p_{f}\right)\left(1-p_{r}\right)}$, where $p_{f}$ and $p_{r}$ are the loss probabilities of the link in the forward and reverse directions, respectively. Each node can measure the loss rate of its links to and from its neighbors by broadcasting probe packet periodically [44][53].

3. The packet queue length. This metric is used to demonstrate the residual buffer space of the node. Each node can get the packet queue length by checking the status of the buffer.

4. The transmission delay of the relay node. In this paper, the delay of the relay node can be got by broadcasting probe packet periodically, which is the same process with the measurement of the link's ETX [44][53], i.e., this metric can be measured in the same process of link's ETX measurement.

5. The distance to the destination node. In this algorithm, since each node equips a GPS device, which can be used to obtain the coordinate of the node, so the distance from the relay node to the destination node can be calculated.

6. The moving speed and direction. The same as the distance to the destination node, the moving speed and moving direction of the node can be got through the GPS device. The moving speed and direction of the candidate nodes are carried by the RREP packets, so when the candidate nodes reply the RREP packets to the source node, the source node can receive the moving speed and direction of the candidate nodes. Since the time needed to send RREP packet from candidate nodes to source node is quite short, so we regard the moving speed and direction that the sender received is up-to-date.

Based on the induction above, the residual energy, the packet queue length, and the moving speed and moving direction of the neighbors are needed to be sent to the sender; the link's ETX, the transmission delay of the neighbors, and the neighbors' distance to the destination node can be calculated or measured by the sender.

It should be noted that the number and kind of the parameters used in this algorithm are not fixed; they can be changed according to the different applications easily and conveniently. This is also the advantage of CBRT, which is flexible without increase the algorithm complexity.
In this section, we compare the performance of CBRT with the traditional opportunistic routing ExOR [20]. The results can be found in Figure 10 to Figure 17. The parameters of the simulation are: deployment area: $1000 \mathrm{~m} \times 1000 \mathrm{~m}$; radio propagation model: two ray ground [44]; initial node transmission range: $500 \mathrm{~m}$; initial energy: $5 \mathrm{~J}$; high transmission power: $0.8 \mathrm{~W}$; low transmission power: $0.1 \mathrm{~W}$; node number 25-150; average moving speed: $0.2 \mathrm{~m} / \mathrm{s}$; receiving power: $0.05 \mathrm{~W}$; maximum allowed packet queue length: 20 packets; traffic type: CBR (constant bit rate); CBR rate: 512 Bytes $\times$ 6 per second [44][53]. Moreover, this simulation mainly concentrates on the network layer and the MAC layer protocol is IEEE 802.11[44][53-55]. In this simulation, the variable is the number of node in the network, i.e., the density of the network ${ }^{1}$.

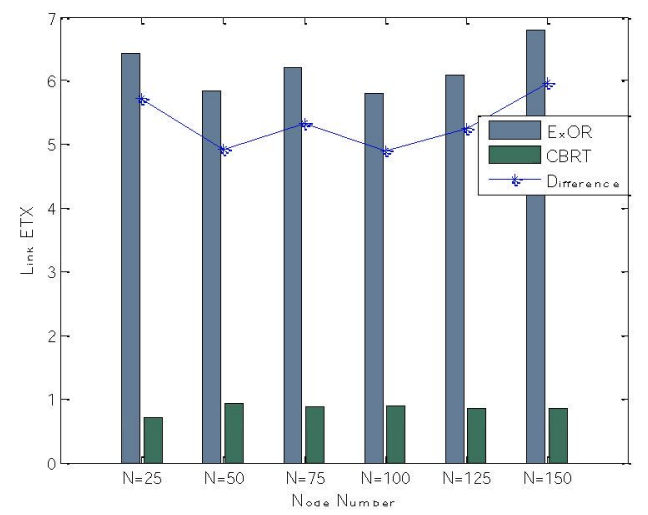

Fig. 10. The ETX of ExOR and CBRT.

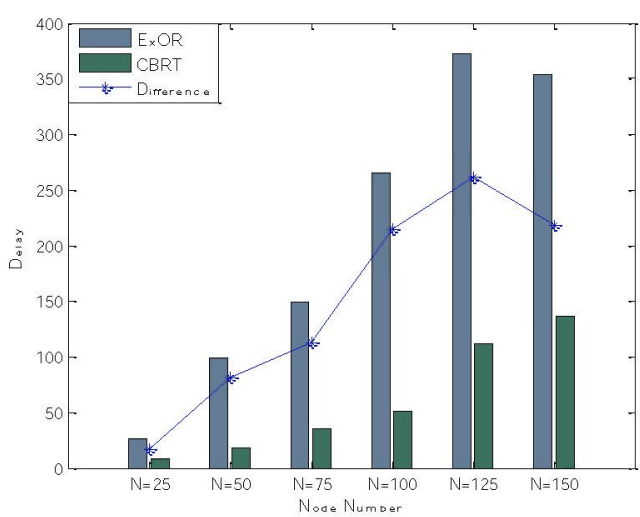

Fig. 11. The transmission delay of ExOR and CBRT.
${ }^{1}$ In this simulation, we only takes the node number as the variable; however, other simulation parameters, such as the traffic load, the transmission power, the moving speed, the congestion, etc., also can affect the results of the simulation; the effect of these parameters will be investigated in the future work. 


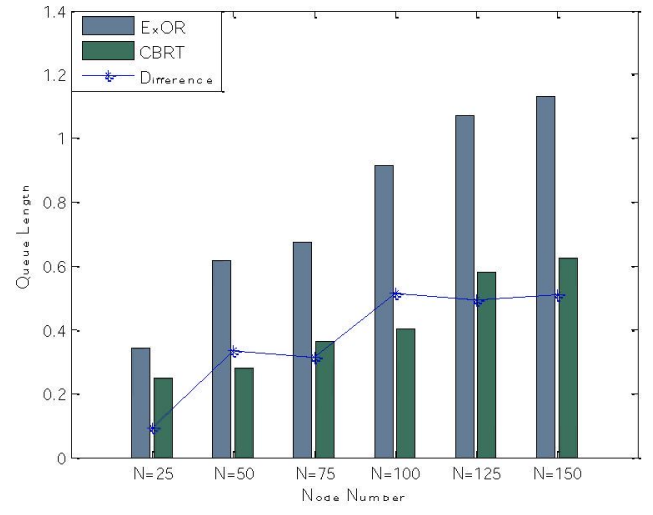

Fig. 12. The packet queue length of ExOR and CBRT.

In Figure 10, the ETX of CBRT and ExOR are compared. From Figure 10, we can find that with the increase of the node number, the ETX in ExOR varies between 6 and 7; however, this value is only 1 in CBRT. This conclusion demonstrates that in ExOR, the ETX is about 6 times larger than that in CBRT. In MANETs, small ETX means small transmission delay, which can be found in Figure 11. In Figure 11, with the increase of the node number, both the transmission delays in CBRT and ExOR increase. However, the increase in ExOR is more serious than that in CBRT. This is due to the high ETX in ExOR. Not only the high ETX but also the packet queue length of the node can increase the transmission delay. As shown in Figure 12, the packet queue length in ExOR is also larger than that in CBRT. With the increase of the node number, the packet queue length increases both in ExOR and CBRT algorithm. However, the packet queue length increases faster in ExOR than CBRT. The Figure 11 and Figure 12 can be explained by each other: the large packet queue length means large transmission delay; conversely, the large transmission delay causes the increase of the packet queue length. Moreover, as shown in Figure 10 and Figure 12, large ETX and packet queue length deteriorate the performance of transmission delay in ExOR.

The Figure 10, Figure 11 and Figure 12 demonstrate that the network performance has been improved greatly by CBRT. As shown in Figure 10, Figure 11, and Figure 12, the parameters in the network are not alone; they relate to each other and can affect each other. Therefore, the more cross-layer parameters are taken into account, the better network performance is. Since the CBRT have the ability to handle multi-parameters without increasing the complexity of the algorithm, so the CBRT is more effective than the traditional opportunistic routing algorithm.

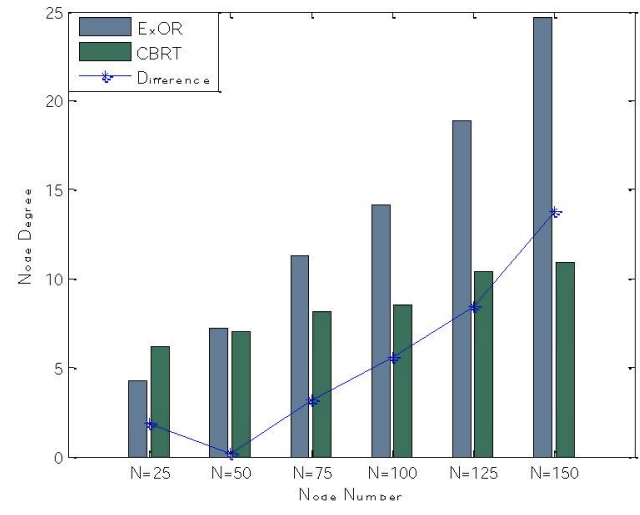

Fig. 13. The node degree of ExOR and CBRT.

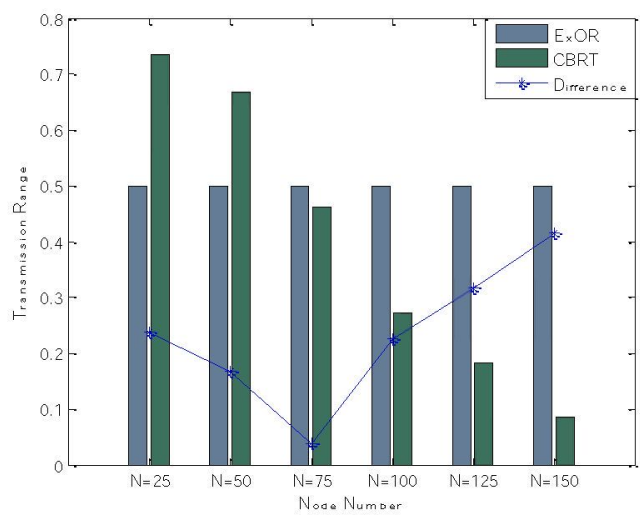

Fig. 14. The transmission range of ExOR and CBRT.

As shown in Figure 13, due to the topology control in CBRT, the RND in CBRT varies between 6 and 10, which is in the healthy region that we define. In Figure 13, when the node number increases, due to the lack of topology control in ExOR, the node degree in ExOR varies greatly. For instance, when the node number is 150, the node degree in ExOR is about 5 times larger than that when the node number is 25 . The reason is that the transmission power is constant in ExOR, so when the node number increases, the node degree will increase greatly. This also means that the packet delivery ratio varies greatly in ExOR. However, due to the topology control in CBRT, when the node number increases, for maintaining stable RND in the candidate relays set, the transmission power will be adjusted according to the value of RND, which can be found in Figure 14. In Figure 14, when the node number increases, due to the OTC algorithm, the transmission power of CBRT decreases to maintain stable RND in the candidate relays set, i.e. the more nodes in the network, the smaller transmission range is. So in Figure 13, the RND in CBRT algorithm various slightly, which is in the region that we set in Section 4 . 


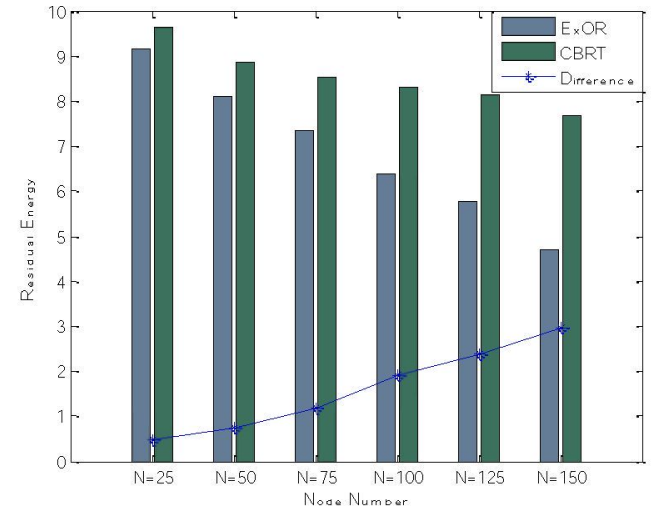

Fig. 15. The residual energy of ExOR and CBRT.

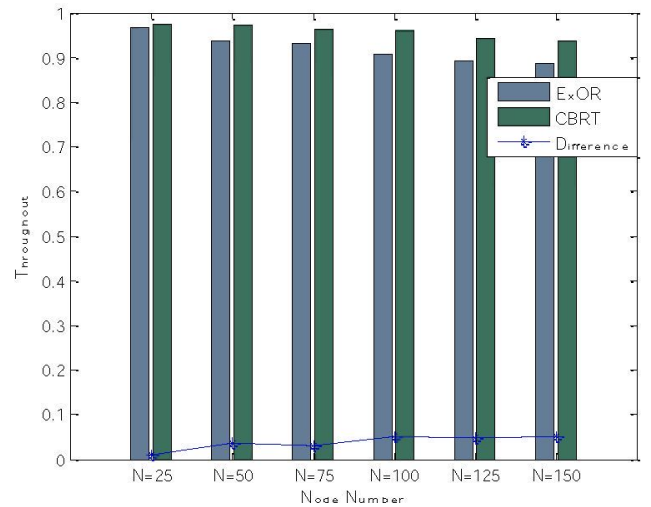

Fig. 16. The through of ExOR and CBRT.

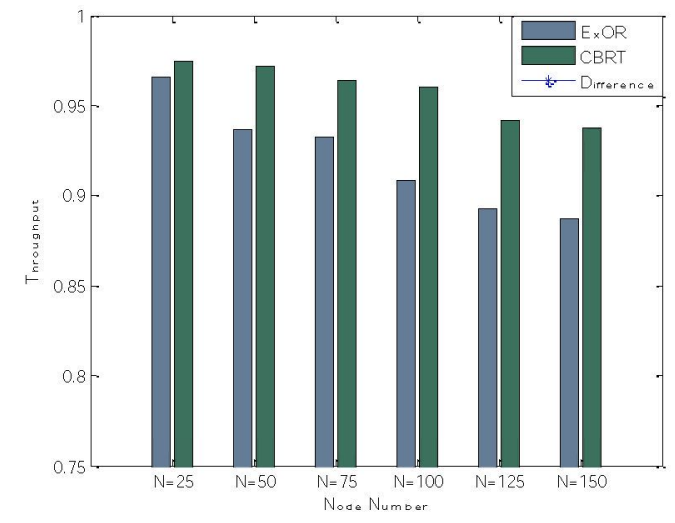

Fig.17. The enlarged view of Fig. 16.

Another advantage of CBRT is the energy consumption. As shown in Figure 15, the residual energy in CBRT is larger than that in ExOR. With the increase of the node number, the residual energy decreases both in ExOR and CBRT. However, the energy is consumed faster in ExOR than that in CBRT. The excellent performance of energy consumption in CBRT owes to the balanced performance of the cross-layer parameters, which can be found in Figure 10, Figure 11, Figure 12, and Figure 14. The excellent performance of link's ETX, transmission delay, packet queue length, and transmission power in CBRT deduces the energy consumption greatly. Similar to the residual energy, in Figure 16 and Figure 17, with the increasing of the node number, the throughout both in ExOR and CBRT will decrease; moreover, the decreasing in
ExOR is faster than that in CBRT. This is also because of the balanced performance of cross-layer parameters in CBRT. In Figure 16, the throughputs of CBRT under different node number are seems the same; however, they are different in fact. This is because the differences between these throughputs are slight, so they are similar. For explain this clearly, we drew the enlarged view of Figure 16 in Figure 17. In Figure 17, we can find that the throughputs under different node number are different; and with the increase of the node number, the throughputs reduce.

As shown in Figure 18, the only disadvantage of CBRT is that with the increase of the node number, the link lifetime in CBRT decreases; however, the link lifetime varies slightly in ExOR. This can be explained by Figure 14. As shown in Section 4, when the moving speed is stable, the larger transmission power, the smaller link lifetime is. Moreover, in CBRT, when the node number increases, the transmission power decreases, which also means small link lifetime. However, since the transmission power is not adjusted in ExOR, so the link lifetime keeps constant.

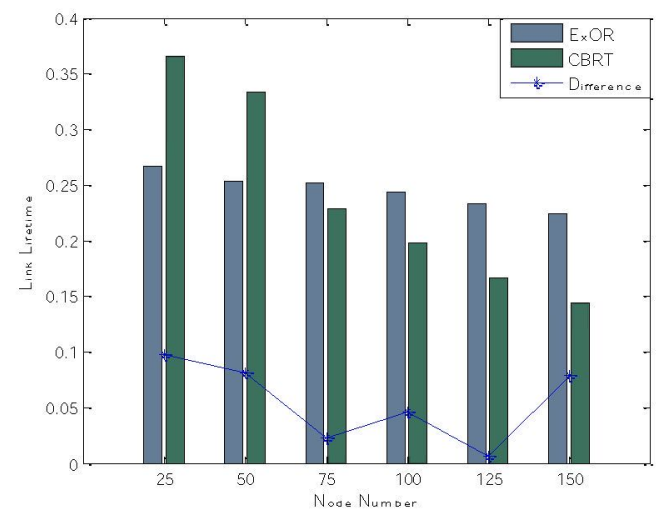

Fig. 18. The residual link lifetime of ExOR and CBRT.

\section{CONCLUSION}

In this paper, we propose the cross-layer and reliable opportunistic routing algorithm (CBRT) for MANETs. In CBRT, the RND in the CRS is a range rather than a constant number. The node is divided into different categories based on the value of RND. The nodes adjust their transmission power according to the RND in CRS. The cross-layer metrics are not inputted into the fuzzy logic system directly; the inputs are the relative variances of these metrics. The calculation of the node utility is weight based. The weight and the priority of the metric are used to calculate the node utility. The relaying node whose node utility is high will have high priority to relay the data packet. By these innovations, the network performance in CBRT is much better than that in ExOR; however, the computation complexity is not increased.

\section{REFERENCES}

[1] F. Cadger, K. Curran, J. Santos, S. Moffett, "A Survey of Geographical Routing In Wireless Ad-Hoc Networks", IEEE Communications Survey and Tutorials, vol. 15, no. 2, 2013, pp: 621-653.

[2] S. Lou, J. He, W. Song, "Effectiveness Analysis of Communications Jamming to Battlefield Ad Hoc Network", in Proceedings of 4th 
International Conference on Computer Engineering and Networks, vol. 355, pp: 1071-1078, Shanghai, China, July, 2014.

[3] S.A. Sultan, M.M.A. Doori, A.H.A. Bayatti, H. Zedan, "A Comprehensive survey on vehicular Ad Hoc network", Journal of Network and Computer Applications, vol. 37, 2014, pp:380-392.

[4] Y.S. Chen, C.S. Hsu, C.H. Cheng, "Network mobility protocol for vehicular ad hoc networks", International Journal of Communication Systems, vol. 27, no. 11, 2014, pp: 3024-3063.

[5] S. Zeadally, R. Hunt, Y.S. Chen, A. Irwin, A. Hassan, "Vehicular ad hoc networks (VANETS): status, results, and challenges", Telecommunication Systems, vol. 50, no. 4, 2012, pp: 217-241.

[6] S. Lu, Z. Wang, Z. Wang, S. Zhou, "Throughput of Underwater Wireless Ad Hoc Networks with Random Access: A Physical Layer Perspective", IEEE Transactions on Wireless Communications, vol. 14, no. 11, 2015, pp: 6257-6268.

[7] A. Mora, D. Ganger, G. Wells, J. Zhang, "Ad-hoc multi-hop underwater optical network for deep ocean monitoring", 2013 OCEANS, San Diego, USA, September, 2013, pp: 1-5.

[8] A. Patil, M. Stojanovic, "A node discovery protocol for ad hoc underwater acoustic networks". Wireless Communications and Mobile Computing, vol. 13, no. 3, pp: 277-295.

[9] S. Biswas, R. Morris, "ExOR: opportunistic multi-hop routing for wireless networks," in Proceedings of the 2005 SIGCOMM, Philadelphia, USA, August 2005, pp: 133-144.

[10] S. Biswas, R. Morris, "Opportunistic routing in multi-hop wireless networks", ACM SIGCOMM computer Communication Review, vol. 34, no. 1, 2004, pp: 69-74.

[11] H. Liu, B. Zhang, H.T. Mouftah, X. Shen, "Opportunistic routing for wireless ad hoc and sensor networks: Present and Future directions", IEEE Communications Magazine, vol. 47, no. 12, 2009, pp: 103-109.

[12] X. Mao, S. Tang, X. Xu, X.Y. Li, H. Ma, "Energy-Efficient Opportunistic Routing in Wireless Sensor Networks", IEEE Transactions on Parallel and Distributed Systems, vol. 22, no. 11, 2011, pp: 1934-1942.

[13] N. Chakchouk, "A Survey on Opportunistic Routing in Wireless Communication Networks", IEEE Communications Surveys and Tutorials, vol. 17, no. 4, 2015, pp: 2214-2241.

[14] N. Li, J.F. Martinez, V.H. Diaz, "The Balanced Cross-Layer Design Routing Algorithm in Wireless Sensor Networks Using Fuzzy Logic", Sensors, vol. 15, no. 8, 2015, pp: 19541-19559.

[15] R. Alsaqour, M. Abdelhaq, R. Saed, M. Uddin, O. Alsukour, M. Alhubaishi, T. Alahdal, "Dynamic packet beaconing for GPSR mobile ad hoc position based routing protocol using fuzzy logic", Journal of Network and Computer Applications, vol. 47, 2015, pp: 32-46.

[16] H. Tahen, P. Neamatollahi, O.M. Younis, S. Naghibzadeh, M.H. Yaghmaee, "An energy-aware distributed clustering protocol in wireless sensor network using fuzzy logic", Ad Hoc Networks, vol. 10, no. 7, 2012, pp: 1469-1481.

[17] A.M. Ortiz, F. Royo, T. Olivares, J.C. Castillo, L.O. Barbosa, P.J. Marron, "Fuzzy-logic based routing for dense wireless sensor networks", Telecommunication Systems, vol 52, no. 4, 2013, pp: 2687-2697.

[18] Y.K. Tamandani, M.U. Bokhari, "SEPFL routing protocol based on fuzzy logic control to extend the lifetime and throughput of the wireless sensor network", Wireless networks, vol. 22, no. 2, pp: 647-653.

[19] J.G. Guan, F.R. Yu, S. Jiang, G. Wei, "Prediction-Based Topology Control and Routing in Cognitive Radio Mobile Ad Hoc Networks," IEEE Transactions on Vehicular Technology, vol. 59, no. 9, 2010, pp: 4443-4452.

[20] S. Biswas, R. Morris, "ExOR: Opportunistic Multi-Hop Routing for Wireless Networks", SIGCOMM'05, Pennsylvania, USA, August, 2005, pp: $133-143$

[21] J. Luo, J. Hu, D. Hu, R. Li, "Opportunistic Routing Algorithm for Relay Node Selection in Wireless Sensor Networks", IEEE Transactions on Industrial Informatics, vol. 11, no. 1, 2015, pp: 112-121.

[22] J. Zuo, C. Dong, H.V. Nguyen, S.X. Ng, L.L. Yang, L. Hanzo, "Cross-Layer Aided Energy-Efficient Opportunistic Routing in Ad Hoc Networks", IEEE Transactions on Communications, vol. 62, no. 2, 2014, pp: $522-535$.

[23] L. Cheng, J. Niu, J. Cao, S.K. Das, Y. Gu, "QoS Aware Geographic Opportunistic Routing in Wireless Sensor Networks", IEEE Transactions on Parallel and Distributed Systems, vol. 25, no. 7, 2014, pp: 1864-1875.

[24] M. Xiao, J. Wu, L. Huang, "Community-Aware Opportunistic Routing in Mobile Social Networks", IEEE Transactions on Computers, vol. 63, no. 7, 2014, pp: 1682-1695.
[25] Z. Wang, Y. Chen, C. Li, "CORMAN: A Novel Cooperative Opportunistic Routing Scheme in Mobile Ad Hoc Networks", IEEE Journal on Selected Areas in Communications, vol. 30, no. 2, 2012, pp: 289-296.

[26] X. Mao, S. Tang, X. Xu, X.Y. Li, H. Ma, "Energy-Efficient Opportunistic Routing in Wireless Sensor Networks", IEEE Transactions on Parallel and Distributed Systems, vol. 22, no. 11, 2011, pp: 1934-1942.

[27] T. Jaradat, D. Benhaddou, M. Balakrishnan, A. Al-Fuqaha, "Energy Efficient Cross-Layer Routing Protocol in Wireless Sensor Networks Based on Fuzzy Logic", In proceedings of the 9th international wireless communication and mobile conference, Sardinia, Italy, July 2013, pp: 177-182.

[28] C. Fathy, M.T. El-Hadidi, M.A. El-Nasr, "Fuzzy-based Adaptive Cross Layer Routing Protocol for Delay Sensitive Applications in MANET", In Proceedings of the IEEE International Conference on Communication, Ottawa, Canada, June 2012, pp: 248-253.

[29] I.S. Alshawi, L. Yan, W. Pan, B. Luo, "Lifetime Enhancement in Wireless Sensor Networks Using Fuzzy Approach and A-Star Algorithm", IEEE Sensors Journal, vol. 12, 2012, pp: 3010-3018.

[30] H. Jiang, Y. Sun, R. Sun, H. Xun, "Fuzzy logic based Energy Optimized Routing for Wireless Sensor Networks", International Journal of Distributed Sensor Network, 2013, 2013, pp: 1-8.

[31] S.S. Bhunia, B. Das, N. Mukherjee, "Multi criteria decision alalysis assisted routing in wireless sensor network using fuzzy rules", In Proceedings of the 2015 International Conference on Distributed Computing and Networking, Goa, India, January 2015, pp: 1-7.

[32] H. Viittala, M. Hamalainen, J. Tinatti, "Zone based fuzzy routing for WBANs", In Proceedings of the Internatioal Symposium on Medical Information and Communication Technology, Kamakura, Japan, March 2015, pp: 93-97.

[33] S. Jain, M. Chawla, V.N.G.J. Soares, J.J. Rodrigues, "Enhanced fuzzy logic based spray and wait routing protocol for delay tolerant networks", International Journal of Communication System, 2014, pp:1-25.

[34] R. Jain, S. Garg, "Dynamic source routing protocol for ad hoc network using the concept intelligent agent fuzzy logic", International Journal of Engineering and Management Research, vol. 4, no. 3, 2014, pp:80-86.

[35] A. Singhal, A.K. Daniel, "Fuzzy logic based stable on-demand multipath routing protocol for mobile Ad Hoc network", In Proceedings of the International Conference on Advanced Computing and Communication Technologies. Rohtak, India, February 2014, pp: 421-426.

[36] H. Uster, H. Lin, "Integrated topology control and routing in wireless sensor networks for prolonged network lifetime", Ad Hoc Networks, vol. 2011, no. 9, 2011, pp: 835-851.

[37] Y. Xue, C. Tang, F. Tang, Y. Yang, J. Li, M. Guo, J. Wu, "Primary user activity prediction based joint topology control and stable routing in mobile cognitive networks", In Proceedings of the 2016 IEEE Wireless Communications and Networking Conference, Doha, Qatar, April 2016, pp: 1-6.

[38] M. Jouhar, K. Ibrahimi, M. Benattou, "Topology control through depth adjustment and transmission power control for UWSN routing protocols", In Proceedings of the 2015 International Conference on Wireless Networks and Mobile Communications, Marrakech, Morocco, October 2015, pp: $1-5$.

[39] W. Wong, X. Chen, F. Long, S.H. Gary Chan, "Joint topology control and routing assignment for wireless mesh with directional antennas", In Proceedings of the 2012 IEEE Global Communications Conference, California, USA, December 2012, pp: 1-6.

[40] L. Ding, W. Wu, J. Willson, H. Dou, W. Lee, D-Z. Du, "Efficient Algorithms for Topology Control Problem with Routing Cost Constraints in Wireless Networks", IEEE Transactions on Parallel and Distributed Systems, vol. 11, no. 10, 2011, pp: 1601-1609.

[41] Q. Guan, F.R. Yu, S. Jiang, G. Wei, "Prediction-Based Topology Control and Routing in Cognitive Radio Mobile Ad Hoc Networks", IEEE Transactions on Vehicular Technology, vol. 59, no. 9, 2010, pp: 4443-4452.

[42] L. Chen, Q. Zhang, M. Li, W. Jia, "Joint Topology Control and Routing in IEEE 802.11-Based Multiradio Multichannel Mesh Networks", IEEE Transactions on Vehicular Technology, vol. 56, no. 5, 2007, pp: 3123-3136

[43] K. Hadawale, S. Barve, "Link prediction-based topology control and adaptive routing in Cognitive Radio Mobile Ad-Hoc Networks", In Proceedings of the 2014 IEEE Global Conference on Wireless Computing and Networking, Lonavala, India, December 2014, pp: 1-5.

[44] X.M. Zhang, F.F. Zou, E.B. W, D.K. Sung, "Exploring the Dynamic Nature of Mobile Nodes for Predicting Route Lifetime in Mobile Ad Hoc 
Networks", IEEE Transactions on Vehicular Technology, vol. 59, no. 3, 2010, pp: 1567-1572.

[45] R. Sanchez-Iborra, M.D. Cano, "JOKER: A Novel Opportunistic Routing Protocol," IEEE Journal on Selected Areas in Communications, vol. 34, no. 5, 2016, pp: 1690-1703.

[46] R.W.L. Coutinho, A. Boukerche, L.F.M. Vieira, A.A.F. Loureiro, "Transmission Power Control-based Opportunistic Routing for Wireless Sensor Network," in Proceedings of MSWiM'14, Motreal, Canada, September 2014, pp: 219-226.

[47] C. Bettstetter, "On the minimum node degree and connectivity of a wireless multihop network", In Proceedings of the $3 \mathrm{rd}$ ACM International Symposium on Mobile Ad Hoc Networking and Computing, Lausanne, Switzerland, June 2002, PP: 80-91.

[48] F. Xue, P.R. Kumar, "The number of neighbor needed for connectivity of wireless networks," Wireless network, vol. 10, no. 2, 2004, pp: 169-181.

[49] Y. Huang, J.F.M. Ortega, J.S. Pons, L.L. Santidrian, "The influence of communication range on connectivity for resilient wireless sensor networks using a probabilistic approach," International Journal of Distributed Sensor Network, vol. 2013, 2013, pp: 1-11.

[50] Y. Huang, J.F.M. Ortega, V.H. Diaz, J. Sendra, "A novel topology control approach to maintain the node degree in dynamic wireless sensor network," Sensors, vol. 14, no. 3, 2014, pp: 4672-4688.

[51] C. Ai, F. Zheng, et al., "Research on energy monitoring method for wireless sensing network," Automation Instrumentation, vol. 28, no. 12, 2007, pp: 5-7.

[52] X. Jiang, P. Dutta, D. Culler, I. Stoica, "Micro power meter for energy monitoring of wireless sensor network at scale," in Process of 6th IEEE International conference on information processing in sensor networks, Cambridge, USA, 2007, pp: 186-195.

[53] E. Rozner, J. Seshadri, Y.A. Mehta, L. Qiu, "SOAR: simple opportunistic adaptive routing protocol for wireless mesh networks," IEEE Transactions on Mobile Computing, vol. 8, no. 12, 2009, pp: 1622-1635.

[54] D. Huang, "Unlinkability measure for IEEE 802.11 based MANETs," IEEE Transactions on Wireless Communications, vol. 7, no. 3, 2008, pp: 1025-1034.

[55] E. Egbogah, L. Shi, A. Fapojuwo, "Optimal hidden node area for enhancing routing protocol performance in IEEE 802.11 multihop MANETs," Journal of Electrical and Computer Engineering, vol. 2011, 2011, pp: 1-7.

[56] L. Qin, T. Kunz, "Pro-active route maintenance in DSR," ACM SIGMOBILE Mobile Comput. COmmun. Rev., vol. 6, no. 3, 2002, pp: 79-89.

[57] W. Su, S.J. Lee, M. Gerla, "Mobility prediction and routing in ad hoc wireless networks," Int. J. Netw. Manage., vol. 11, no. 1, 2001, pp: 3-30.

[58] P. Samar, S.B. Wicker, "On the behavior of communication links of a node in a multi-hop mobile environment," in Proc. Int. Symp. Mobile ad hoc Netw. Comput. 2004, pp: 145-156.

[59] X. Wu, H.R. Sadjadpour, J.J. Garcia-Luna-Aceves, "An analytical framework for the characterization of link dynamics in MANETs," in Proc. IEEE Mil. Commun. Conf., 2006, pp: 1-7. 